\title{
Fault Detection of a Wheelset Bearing Based on Appropriately Sparse Impulse Extraction
}

\author{
Jianming Ding, Fenglin Li, Jianhui Lin, Bingrong Miao, and Lu Liu \\ State Key Laboratory of Traction Power, Southwest Jiaotong University, Chengdu 610031, China \\ Correspondence should be addressed to Jianming Ding; fdingjianming@126.com
}

Received 16 November 2016; Revised 23 February 2017; Accepted 28 February 2017; Published 18 June 2017

Academic Editor: Mariano Artés

Copyright (c) 2017 Jianming Ding et al. This is an open access article distributed under the Creative Commons Attribution License, which permits unrestricted use, distribution, and reproduction in any medium, provided the original work is properly cited.

\begin{abstract}
Convolution sparse representation (CSR) is a novel compressive sensing technique proposed in 2016 and provides an excellent framework for extracting the impulses induced by bearing faults and the unevenness of wheel tread. However, its sparsity performance on extracting impulses is sensitive to the improper penalty parameter. So, a novel fault detection method, appropriately sparse impulse extraction, is proposed based on the combination of CSR, estimating the number of atom types (ENA), and crest factor. The type of atoms embedded in vibration signals is estimated by ENA. Aiming at the different types of atoms, the impulses with different sparse characteristic are spanned by CSR with different penalty parameters. The appropriately sparse impulses are selected for fault detection based on the maximal crest factor. The simulation validation, experiment verification, and practical application are conducted to validate the effectiveness of the proposed appropriately sparse impulses extraction. These results show that the proposed appropriately sparse impulse extraction not only can obtain fault-characteristic frequency and its harmonics for fault judgment but also describes the dynamic behaviour between elementary defects and their matching surfaces. In addition, the proposed appropriately sparse impulse extraction can isolate the impulses with different types of atoms and is very suitable for detecting the wheelset bearing faults.
\end{abstract}

\section{Introduction}

A wheelset bearing is one of crucial mechanical components in a high-speed train and plays an important role in load bearing, power transmission, and motion transform. During the long-term running process of high-speed train, the sustainable effects of the heavy loads and alternating stresses on the components of wheelset bearing easily lead to the bearing fault initiation (such as spalls, pitting, and burningoff). Once these faults exist in the wheelset bearing, they become increasing worse and finally endanger the service safety of high-speed train [1-3]. Therefore, it is of great significance to detect wheelset bearing faults for ensuring the operational safety and realizing the condition-based maintenance of high-speed train.

Acceleration-based vibration monitoring is a feasible and effective route to detect wheelset bearing faults because of the convenient installation of the measured sensors and the low cost of collecting vibrational signals $[4,5]$. The vibrational signals carry the fruitful information about the working status of the bearing. Once the bearing fault appears, the vibrational signals contain impulse sequences with the rotation of wheelset bearing [6]. The impulses are the essential symptom for bearing faults [7]. So, how to extract the impulses from the vibrational signals using an advanced signal proceeding technique becomes a key of detecting bearing faults. Many advanced signal processing methods are proposed, such as enhanced wavelet transforms $[8,9]$ and modified empirical model decomposition [10].

Compressed sensing (CS) that emerged in 2006 is a new method to extract impulses from the measured vibration signals [11]. The core theory about CS consists of sparse representation and dictionary design. Sparse representation models the analyzed signals as the linear combination of basic elements or atoms in a redundant dictionary. Dictionary design is adapted to the self-feature of the analyzed 


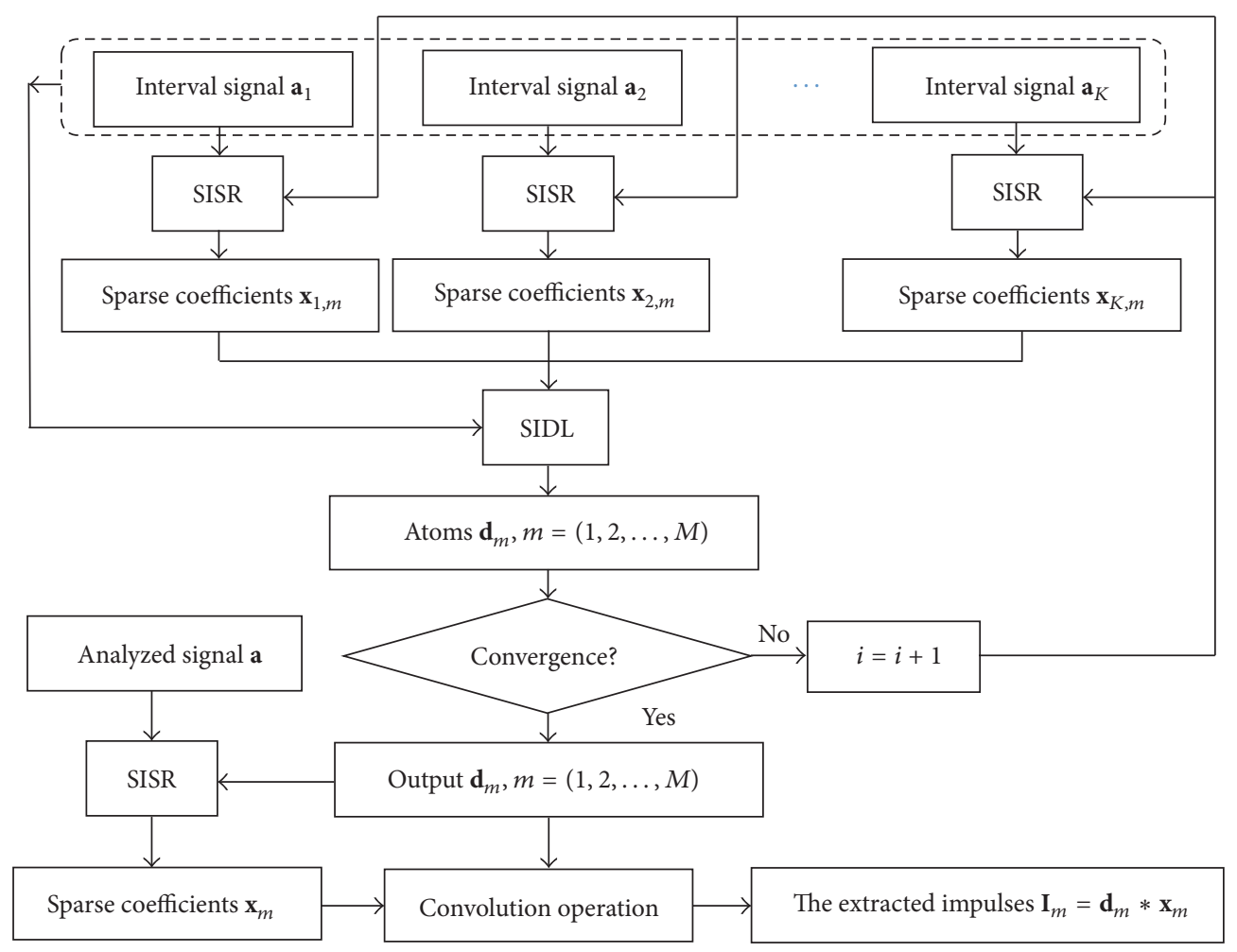

FIGURE 1: The procedure of extracting the impulses.

signals to well match high-level structures embedded in the analyzed signals, as much as possible. CS has been widely applied and generates the state-of-the-art results in learning, neuroscience, signal processing, image and audio processing, classification, and statistics [12-14].

In terms of sparse representation, its extract resolution is an NP-hard problem [15]. Thus, the approximate solutions based on the pursuit algorithms are considered instead. The pursuit algorithms mainly include the greed-based matching pursuit and the convex optimization-based basis pursuit. Matching pursuits are very simple via selecting the dictionary atoms sequentially by the similar measure between the analyzed signal and atoms [8], mainly including matching pursuit (MP) [16], orthogonal matching pursuit (OMP) $[17,18]$, block orthogonal matching pursuit (BOMP) [19], compressive sampling matching pursuit (COSAMP) [20], and subspace pursuit (SSMP) [21]. The resolution methods of basis pursuit have been developed in recent years, mainly including the gradient projection method (GP) [22], sparse reconstruction by separable approximation (SPARSA) [23], iterative thresholding method (ITS) [24], fast iterative thresholding method (FIST) [25], coordinate gradient descent method (CGD) [26], and Bregman iterative algorithm (BIT) [27]. In fact, matching pursuit and basis pursuit share the common the sparse solutions under some favorable conditions [28]. In general, basis pursuit is computationally tractable and not combinational algorithm compared to matching pursuit [29]. So basis pursuit is more suitable for processing large-scale and high-dimension engineering data.
As for dictionary design, the route to designing a dictionary mainly includes manually predefined dictionary (also called the implicit dictionary) and dictionary learning (also called explicit dictionary). The predefined dictionary is designed via the signal transform techniques (such as Dirac, Heaviside, Fourier transform, wavelet transform, or merging mentioned dictionaries to create mega-dictionary) [30]. Dictionary learning is that a dictionary is directly inferred from the measured signals by machine learning technique, mainly including regular dictionary learning (such as optimal directions (MOD, RSDL) [31], K-SVD [32], online dictionary learning [33], and iterative LS-based dictionary learning (ILS-DLA) [34]) and shift-invariant dictionary learning (Lagrange dual method and shift-invariant K-SVD, explicit shift-invariant dictionary learning) [35-37]. Shift-invariant dictionary learning (SIDL) is an extension of regular dictionary learning (RDL) and makes each atom replicated and shifted at each time offset within the signal and more suitable for designing the dictionary to process the signals with the circular modes. Dictionary learning can adaptively fit high-level structures of the analyzed signals and generates more sparse resolution compared to the predefined dictionary.

CS-based impulse extraction techniques become increasing popular by virtue of the excellent performances (such as flexibility, sparsity, and superresolution). The CS based on the combination of matching pursuit and predefined dictionary is used to extract impulses induced by the rotational machine faults for bearing and gear fault detection [38, 39]. The CS 
based on the combination of basis pursuit and predefined dictionary is employed to capture the impulse patterns for rotational machine fault diagnosis [40-42]. The performance of CS on detecting fault outperforms the wavelet transform and empirical model decomposition [38-42]. Because dictionary learning has the advantages and potential on mining high-level structures embedded in vibration signals over the predefined dictionary, SIDL-based CS and basis pursuit is used to extract the circular impulses submerged in the vibration signals of rotating machine system [4346].

Convolution sparse representation (CSR) based on alternating direction method of multiplier (ADMM) is proposed in 2016 as a novel research production of CS [47]. It realizes not only SIDL but also shift-invariant sparse representation (SISR) of analyzed signals. The strategy of the interleaved optimization between SIDL and SISR, instead of the alternating optimization between SIDL and basis pursuit in the traditional SIDL, leads to higher computational efficiency and accuracy. CSR is introduced into the field of wheelset bearing fault detection in this paper. Although CSR has the potential on extracting the impulses embedded in vibration signals, its performance on extracting impulses is sensitive to the improper penalty parameter. When the penalty parameter is very large, the extracted impulses are so sparse that the number of the extracted impulses is far less than the true number of the impulses induced by bearing faults. As a result, this easily leads to the loss of the impulses containing fruitful fault-information. Conversely, the number of the extracted impulses is far more than the true number of the impulses. Then, the true impulses induced by faults are contaminated by the extra false impulses which have adverse influence on detecting faults. The impulses with different sparse characteristic are extracted by CSR with different penalty parameters. An impulse indictor is regarded as a criterion to select the impulses with the properly sparse characteristic for fault judgment. The impulse indictors mainly include kurtosis [48], sparsity [49], permutation entropy [50], infogram [51], and crest factor [52]. Those indictors (e.g., kurtosis, sparsity, and permutation entropy) can only indicate impulse existence but cannot characterize the repetitive characteristic of the impulses [52]. However, the repetitive characteristic of impulses is closely related to their sparse characteristic. So, crest factor of the impulse envelope spectra is regarded as a measured criterion to select the impulses with the properly sparse characteristic in this paper. Meanwhile, CSR cannot learn the number of atoms embedded in vibration signals. So a novel fault detection method, appropriately sparse impulse extraction based on the combination of CSR, crest factor, and ENA, is proposed in this paper.

This paper is organized as follows. The basis theory of the appropriately sparse impulse extraction is introduced in Section 2. Simulation validation is executed in Section 3. Experiment verifications are conducted in Section 4. Section 5 represents the practical application of the proposed method. Section 6 concludes the paper.

\section{Basis Theory of the Appropriately Sparse Impulse Extraction}

2.1. Extracting Impulses with Different Sparse Characteristic Using CSR. Convolution sparse representation (CSR) models an analyzed signal as the sum of a set of convolutions of atoms with the sparse coefficients and is defined as

$$
\begin{aligned}
\left\{\mathbf{d}_{m}, \mathbf{x}_{k, m}\right\}= & \underset{\left\{\mathbf{d}_{m}, \mathbf{x}_{k, m}\right\}}{\arg \min } \frac{1}{2} \sum_{k=1}^{K}\left\|\mathbf{a}_{k}-\sum_{m=1}^{M} \mathbf{d}_{m} * \mathbf{x}_{k, m}\right\|_{F}^{2} \\
& +\lambda \sum_{k=1}^{K} \sum_{m=1}^{M}\left\|\mathbf{x}_{k, m}\right\|_{1},
\end{aligned}
$$

where $\mathbf{a}_{k} \in \mathbb{R}^{n}$ denotes the $k$ th analyzed vibration acceleration signals, $n$ means the length of analyzed signal, $\mathbf{d}_{m} \in \mathbb{R}^{p}$ denotes a set of atoms, $p$ is the length of atom, $*$ means the convolution operation, $\mathbf{x}_{k, m} \in \mathbb{R}^{n-p+1}$ denotes sparse coefficients related to given signal $\mathbf{s}_{k}$ and atom $\mathbf{d}_{m}, K$ is the number of interval signals $\mathbf{s}_{k}, M$ is the number of atoms $\mathbf{d}_{m}$, and $\lambda \in \mathbb{R}^{+}$means penalty parameter which determines the sparse characteristic.

The one inverse problem is that the sparse coefficients $\mathbf{x}_{k, m}$ are resolved by the shift-invariance sparse coding (SISC) of (1), when the signals $\mathbf{a}_{k}$ and atoms $\mathbf{d}_{m}$ are given. The SISC can be expressed as

$$
\begin{aligned}
\mathbf{x}_{k, m}= & \arg \min \frac{1}{2} \sum_{k=1}^{K}\left\|\mathbf{a}_{k}-\sum_{k} \mathbf{d}_{m} * \mathbf{x}_{k, m}\right\|_{F}^{2} \\
& +\lambda \sum_{k=1}^{K} \sum_{m=1}^{M}\left\|\mathbf{x}_{k, m}\right\|_{1} .
\end{aligned}
$$

In (2), the mutual coupling of the different sparse coefficients $\mathbf{x}_{k, m}$ does not exist with respect to different analyzed signals $\mathbf{s}_{k}$ and the sparse coefficients $\mathbf{x}_{m}$ corresponding to signals a can be individually solved by

$$
\mathbf{x}_{m}=\underset{\mathbf{x}_{m}}{\arg \min } \frac{1}{2}\left\|\mathbf{a}-\sum_{k} \mathbf{d}_{m} * \mathbf{x}_{m}\right\|_{F}^{2}+\lambda \sum_{m=1}^{M}\left\|\mathbf{x}_{m}\right\|_{1} .
$$

The atoms $\mathbf{d}_{m}$ are obtained by SIDL of (1), when the signal $\mathbf{a}_{k}$ and sparse coefficients $\mathbf{x}_{k, m}$ are known. SIDL is described as

$$
\mathbf{d}_{m}=\underset{\mathbf{d}_{m}}{\arg \min } \frac{1}{2} \sum_{k=1}^{K}\left\|\mathbf{a}-\sum_{k} \mathbf{d}_{m} * \mathbf{x}_{k, m}\right\|_{F}^{2}
$$

such that $\left\|\mathbf{d}_{m}\right\|=1 \forall m$.

For the details of the algorithms of SISC and SIDL in CSR based on alternating direction method of multipliers (ADMM), one can refer to [47]. Given the analyzed signals a, the interval signals $\mathbf{a}_{k} \in \mathbb{R}^{n}(k=1,2, \ldots, K)$ are obtained so that $\mathbf{a}=\left[\mathbf{a}_{1}^{T}, \ldots, \mathbf{a}_{K}{ }^{T}\right]^{T} \in \mathbb{R}^{n K}(n=1024, K=8$ in this paper). The procedure of extracting impulses from the signals a based on the SISR and SIDL is proposed and shown in Figure 1. 


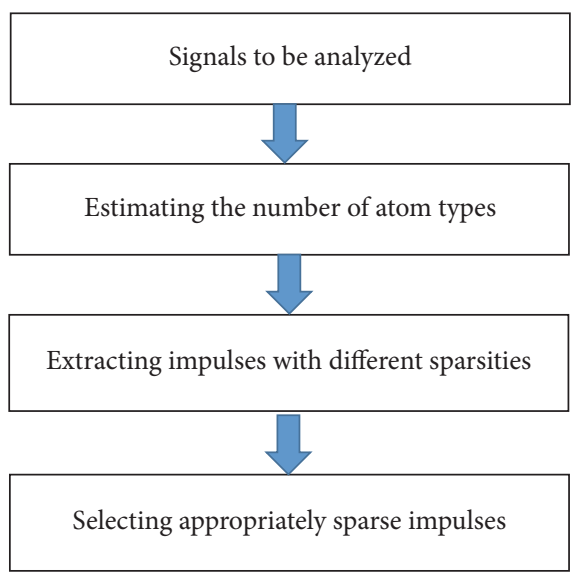

FIGURE 2: Fault detection procedure based on appropriately sparse impulse extraction.

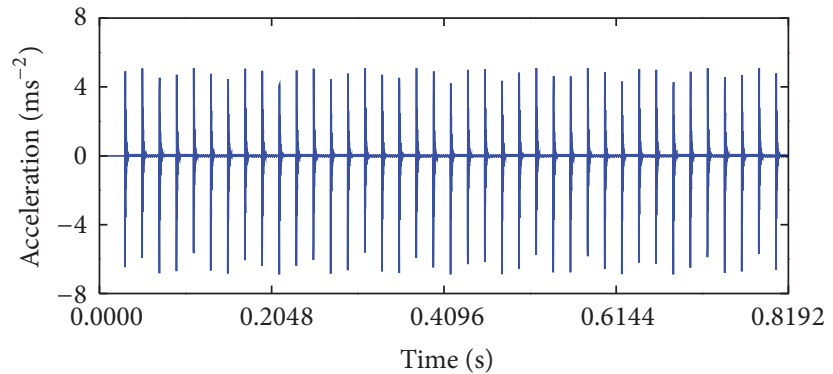

(a)

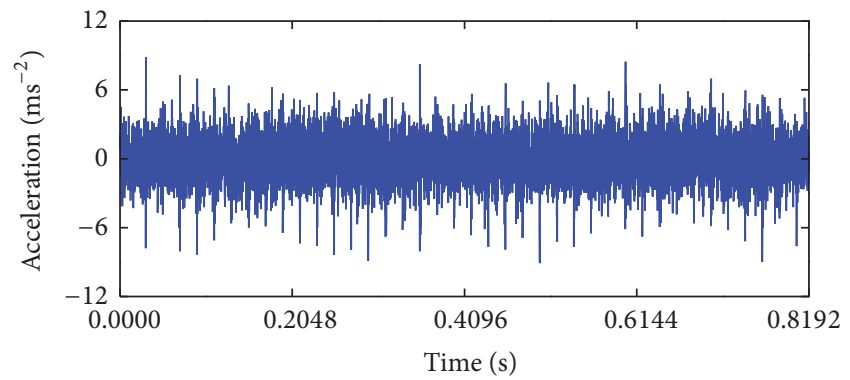

(b)

FIGURE 3: Signals for simulation: (a) the original signals with periodic impulses and (b) the signals with the added white noise (SNR $=-7 \mathrm{~dB}$ ).

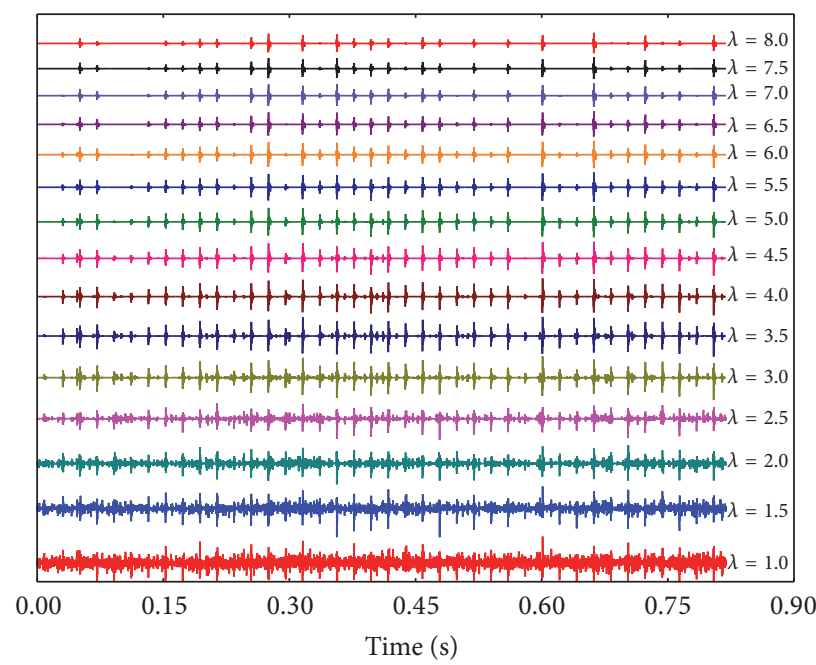

FIGURE 4: The impulses with different sparse characteristic.

In Figure 1, the atoms $\mathbf{d}_{m}$ are learned from the interval signals $\mathbf{a}_{k} \in \mathbb{R}^{n}(k=1,2, \ldots, K)$ through the interleaved iterations of SISC and SIDL of CSR to enhance the learning efficiency. The sparse coefficients $\mathbf{x}_{m}$ associated with the signals $\mathbf{a}=\left[\mathbf{a}_{1}{ }^{T}, \ldots, \mathbf{a}_{K}{ }^{T}\right]^{T} \in \mathbb{R}^{n K}$ are obtained through the SISC based on the learned atoms $\mathbf{d}_{m}$. So, the impulses $\mathbf{I}_{m}$ are extracted by the convolution of atoms $\mathbf{d}_{m}$ and associated sparse coefficients $\mathbf{x}_{m}$ and are expressed as

$$
\mathbf{I}_{m}=\mathbf{d}_{m} * \mathbf{x}_{m} .
$$




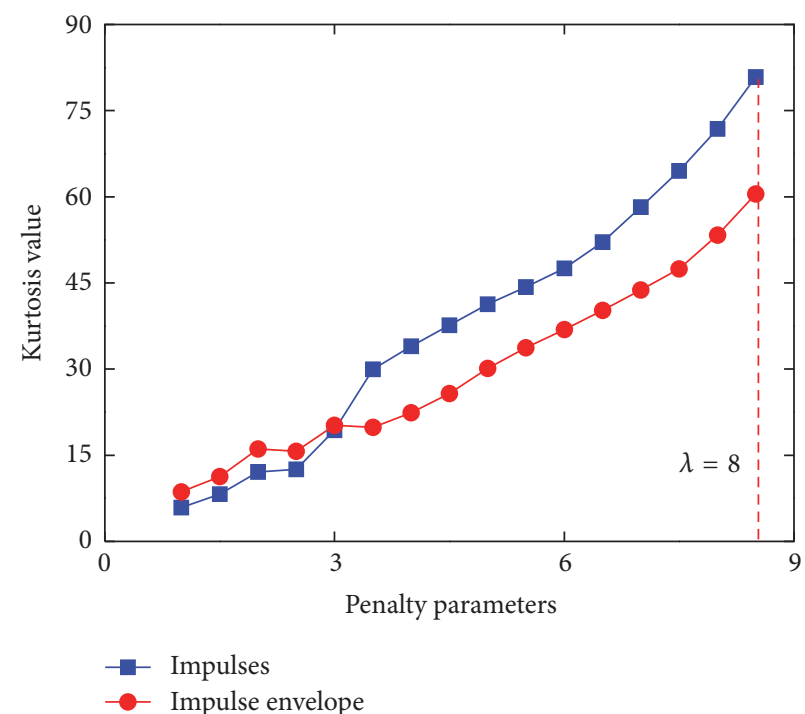

(a)

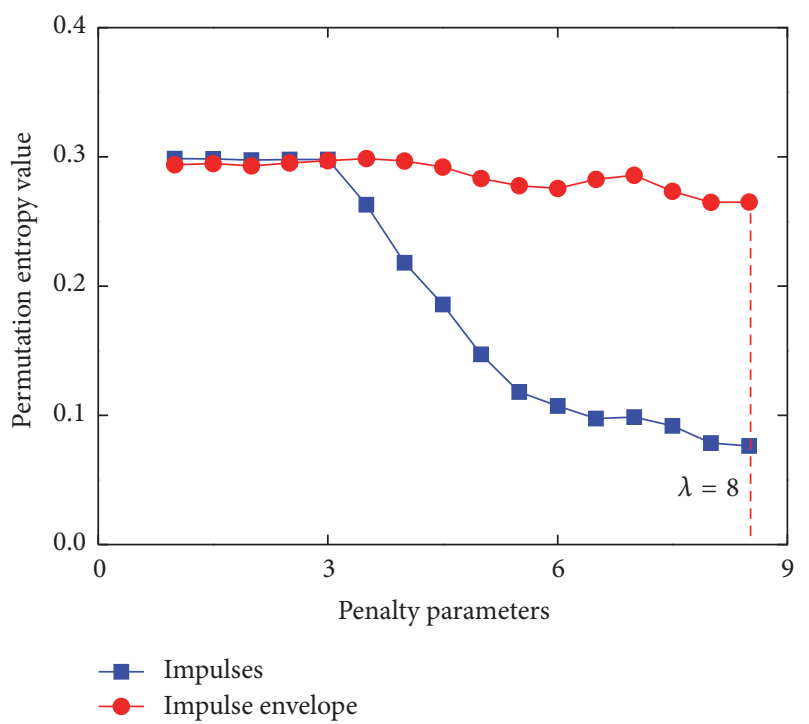

(c)

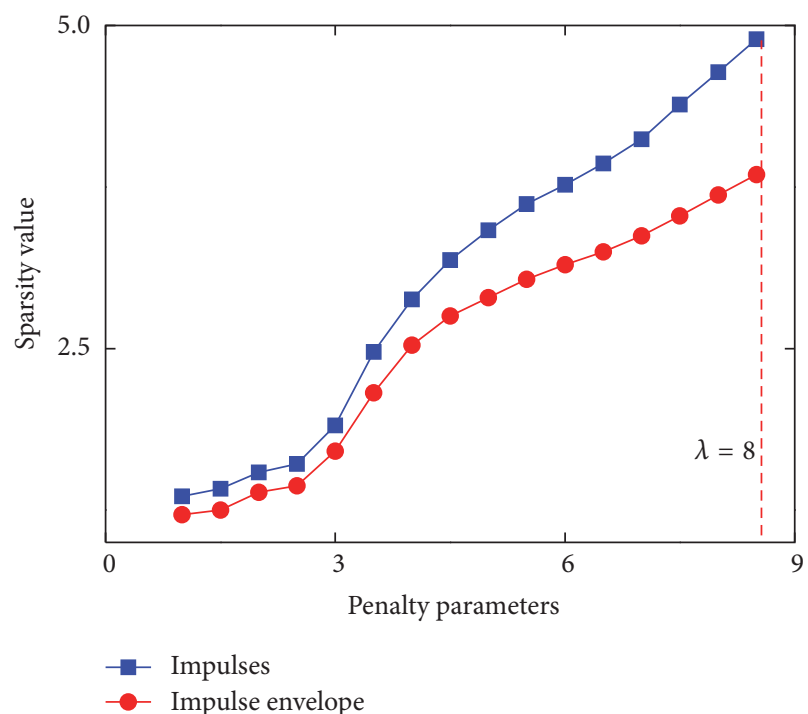

(b)

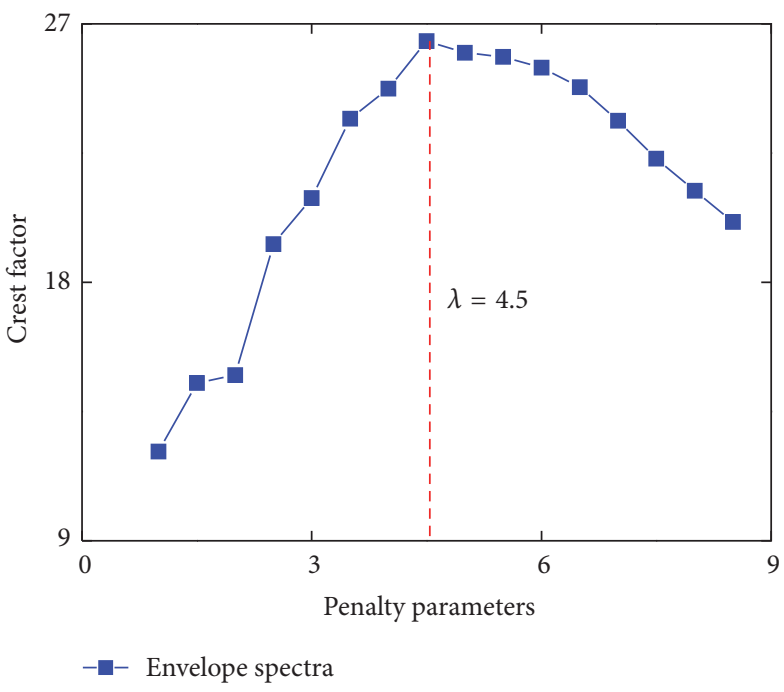

(d)

FIGURE 5: Four indicators for selecting impulses.

The impulses $\mathbf{I}_{m}^{\lambda_{j}}$ with different sparsity characteristic are extracted by CSR with different penalty parameters $\lambda_{j}$, for example,

$$
\mathbf{I}_{m}^{\lambda_{j}}=\mathbf{d}_{m}^{\lambda_{j}} * \mathbf{x}_{m}^{\lambda_{j}} .
$$

In this paper, $\lambda_{j}=\lambda_{1}+0.5(j-1)(j=1,2, \ldots, N)$, $\lambda_{1}=1$, and $N$ is determined when the number of the nonzero elements of sparse coefficients $\mathbf{x}_{m}^{\lambda_{N+0.5}}$ is equal to zero.

2.2. Selection of the Appropriately Sparse Impulses. The Hilbert transform of the extracted impulses $\mathbf{I}_{m}^{\lambda_{j}}$ is expressed as

$$
\mathbf{h}_{m}^{\lambda_{j}}=\frac{2}{\pi} \int_{-\infty}^{\infty} \frac{\mathbf{I}_{m}^{\lambda_{j}}(t)}{t-\tau} d \tau=\frac{1}{\pi t} \mathbf{I}_{m}^{\lambda_{j}}(t)
$$

The envelope spectra $\mathbf{E}_{m}^{\lambda_{j}}$ of the extracted impulses $\mathbf{I}_{m}^{\lambda_{j}}$ are written as

$$
\mathbf{E}_{m}^{\lambda_{j}}=F\left\{\sqrt{\left(\mathbf{I}_{m}^{\lambda_{j}}\right)^{2}+\left(\mathbf{h}_{m}^{\lambda_{j}}\right)^{2}}\right\}
$$

where $F$ denotes Fourier transform.

Crest factor is a measure of a waveform and defined as the ratio of peak values to the effective value. When the sparsity of the extracted impulses is almost identical to the true sparsity of the impulses induced by bearing fault, the fault-characteristic frequency and its harmonics exist in the envelope spectra of the extracted impulses. The higher the number of the periodic impulses is, the larger the peak value of the amplitude about the fault-characteristic frequency 


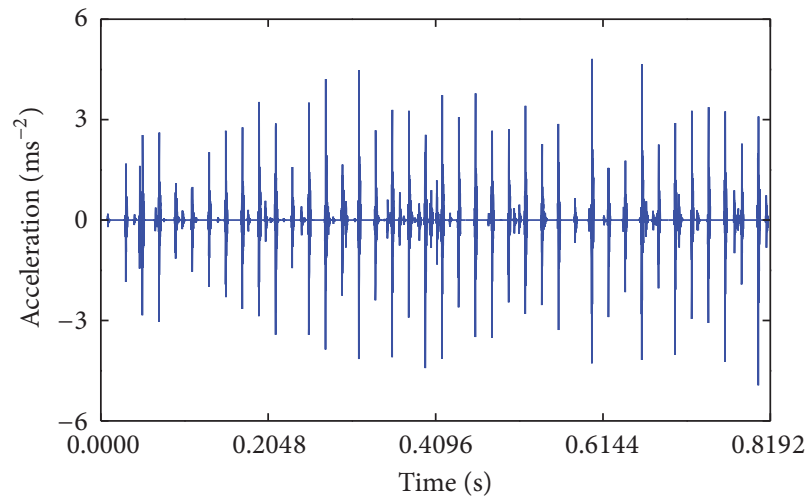

(a)

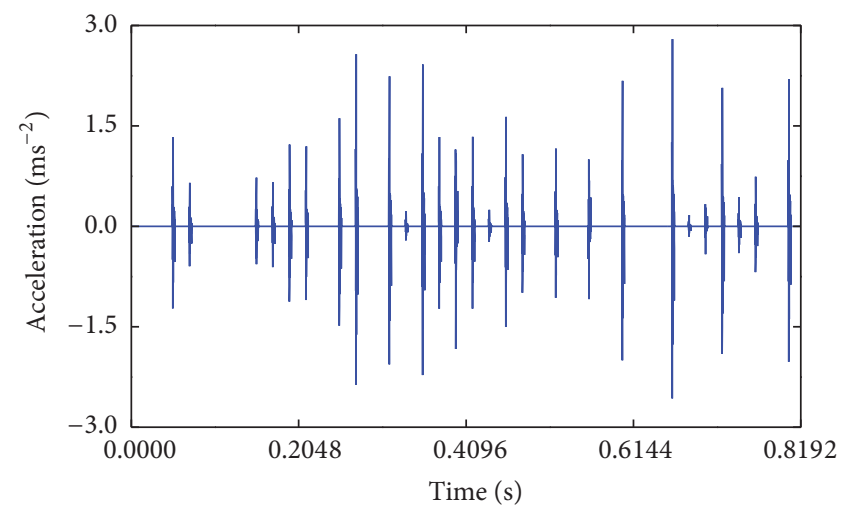

(c)

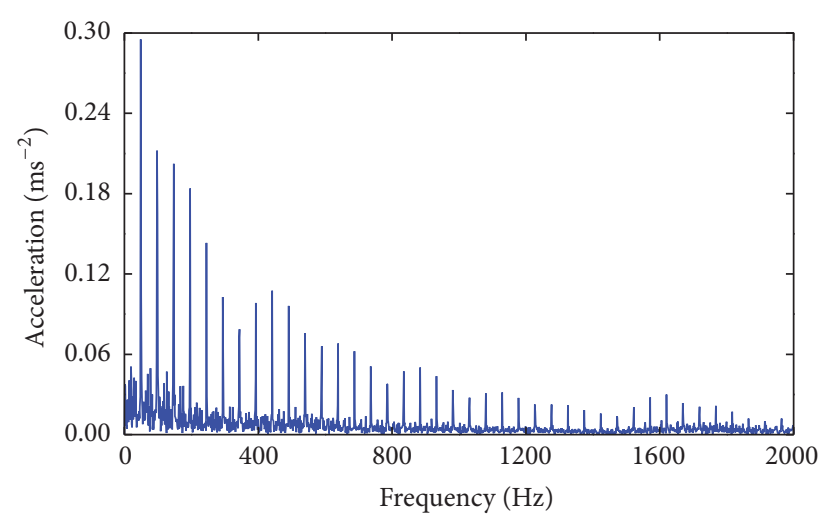

(b)

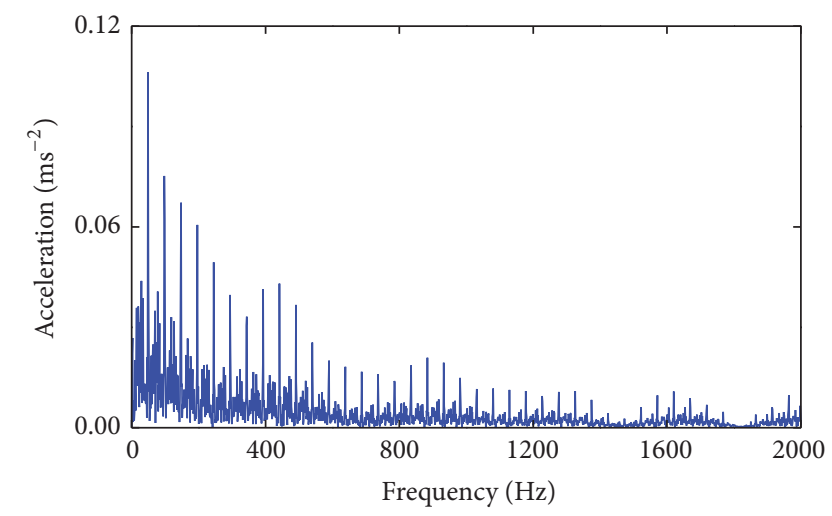

(d)

FIGURE 6: The extracted impulse: (a) by proposed appropriately sparse impulse extraction; (b) the envelope spectra of (a); (c) three other indicators; (d) the envelope spectra of (c).

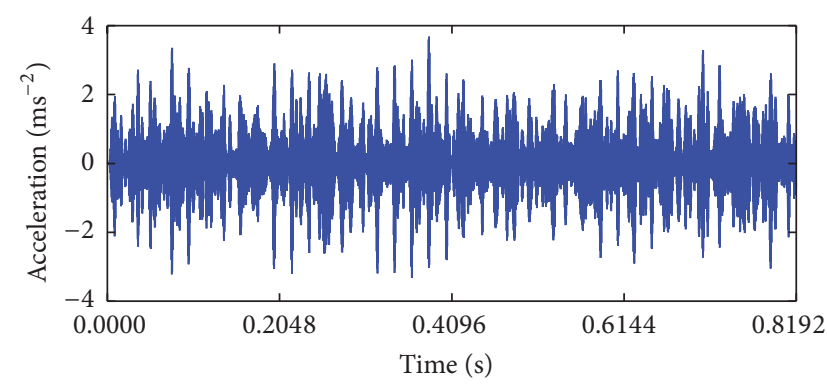

(a)

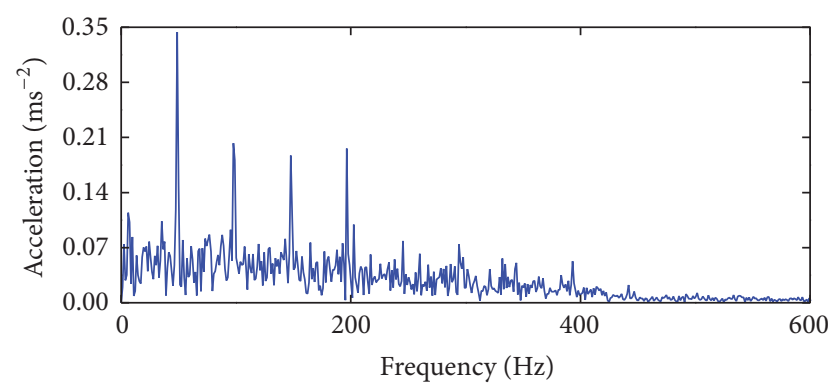

(b)

FIGURE 7: The results obtained by filtering-based demodulation technique: (a) the filtered signals; (b) Hilbert spectra of filtered signals.

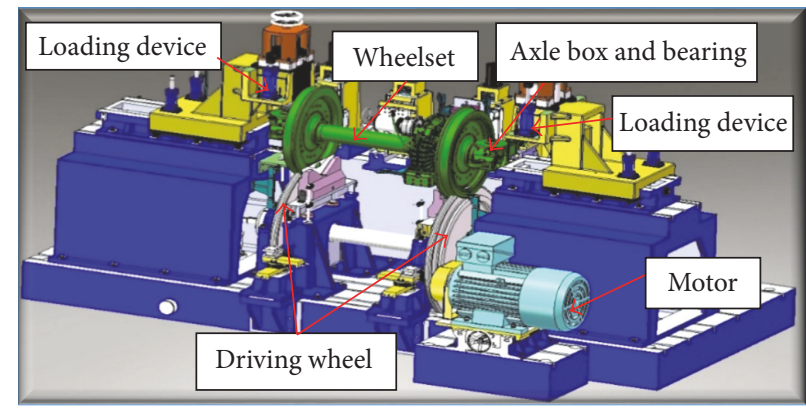

FIGURE 8: Schematic sketch of the wheelset bearing test bench. 


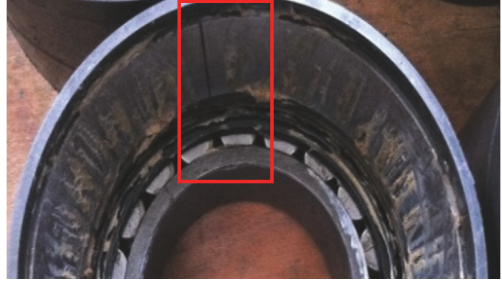

(a)

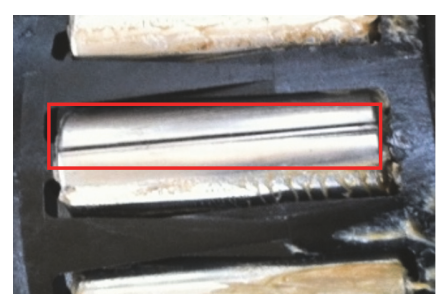

(b)

FIGURE 9: Photo of the artificial faults on the tapered roller and outer race.

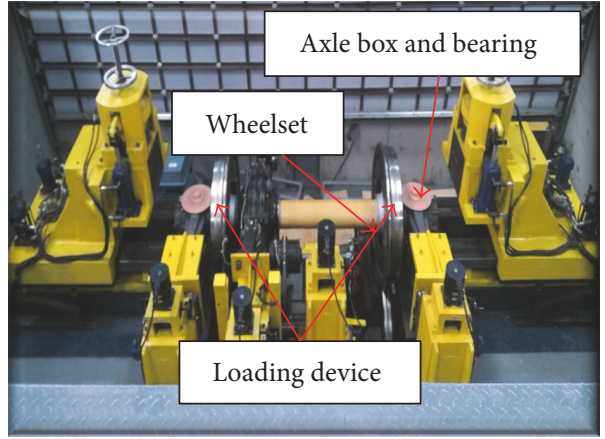

(a)

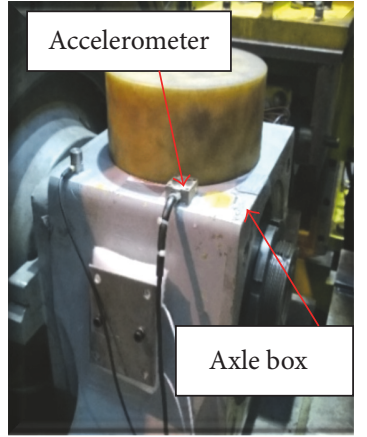

(b)

Figure 10: Photos of the test bench and measurement sensor.

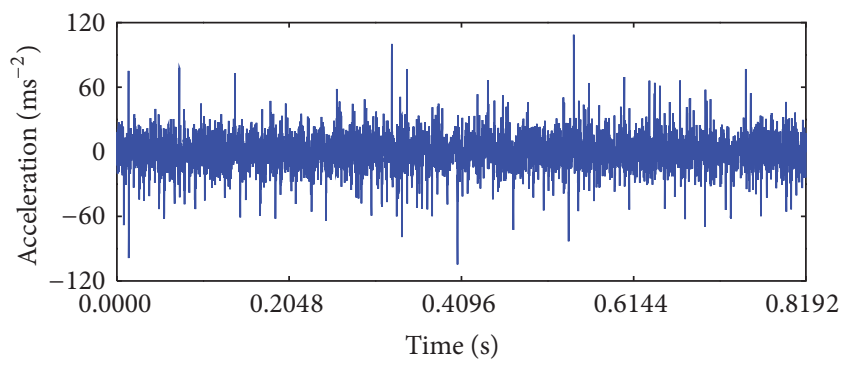

FIGURE 11: The vibration signals of the fault on outer race.

is. The crest factor of envelope spectra $C_{E}^{\lambda_{j}}$ is suitable for characterizing the periodic impulses and expressed as

$$
C_{E}^{\lambda_{j}}=\frac{\max \left(\mathbf{E}_{m}^{\lambda_{j}}(i)\right)}{\sqrt{(1 / N) \sum_{i=1}^{N}\left(\mathbf{E}_{m}^{\lambda_{j}}(i)\right)^{2}}} .
$$

So the maximal crest factor $C$ of envelope spectra is regarded as an indicator to select the approximately sparse impulses.

$$
C=\max \left(C_{E}^{\lambda_{j}}\right), \quad j=(1,2, \ldots, N) .
$$

2.3. Estimating the Number of the Atom Types (ENA). The number of atoms $M$ is not determined from (1) to (3). According to the prior knowledge about the vibration mechanism of impulses induced by bearing faults [5], an impulse can be completely described by two parameters of resonance frequency and damping coefficient. The resonance frequency can identify different atoms. So the dominant frequencies of the learned atoms are used to estimate the number of atoms $M$. The main steps about ENA are expressed as follows.

Step 1. The number $M$ is preset as 4 in wheelset bearing fault detection considering the defects of outer race, inner race, roller, and wheel tread.

Step 2. The atoms of $M$ are obtained by CSR; the dominant frequencies $f_{m}(m=1,2, \ldots, M)$ of each atom are extracted by Fourier transformation.

Step 3. Judge whether the difference of any two dominant frequencies $f_{m}(m=1,2, \ldots, M)$ is more than the frequency resolution $f_{s} p^{-1}$ ( $f_{s}$ denotes the sampling frequency). If so, $M$ is output as the estimated value of the number of atoms; if not, $M=M-1$.

Step 4. Circularly execute Steps 2 and 3 until the difference of any two dominant frequencies is more than frequency resolution.

2.4. Fault Detection Procedure of Appropriately Sparse Impulse Extraction. The fault detection procedure based on the appropriately sparse impulse extraction is proposed and shown in Figure 2. The main steps include the following. 


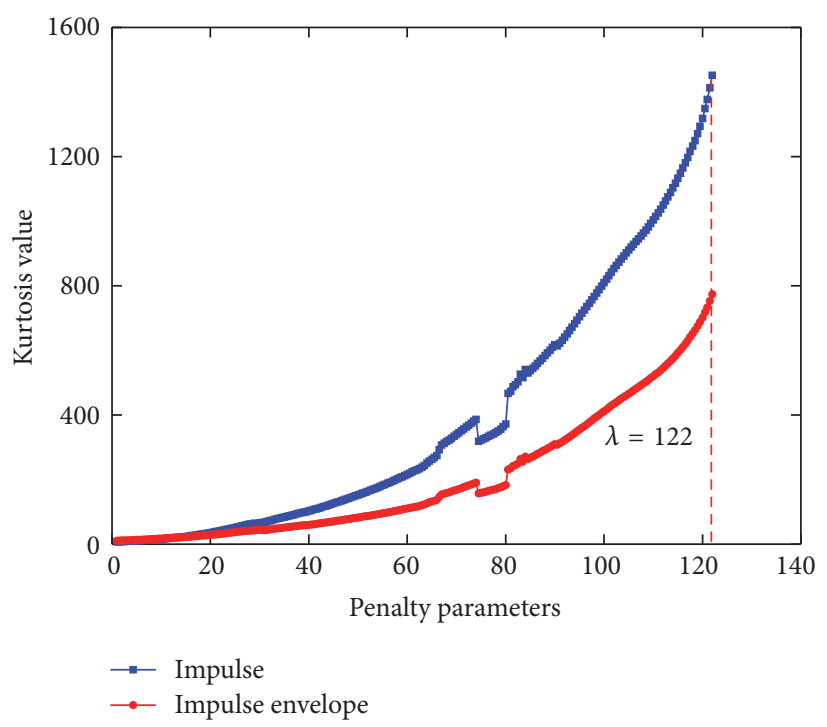

(a)

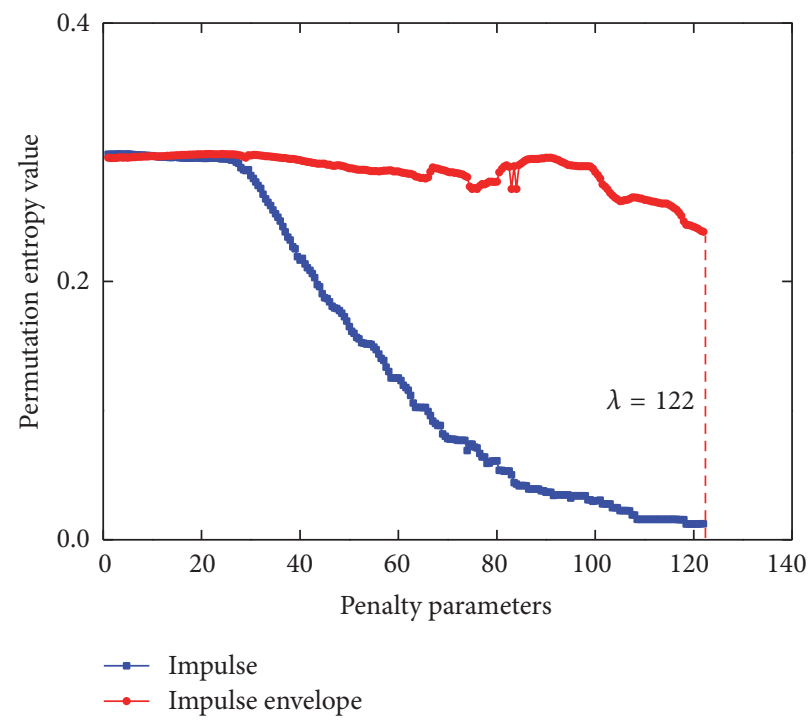

(c)

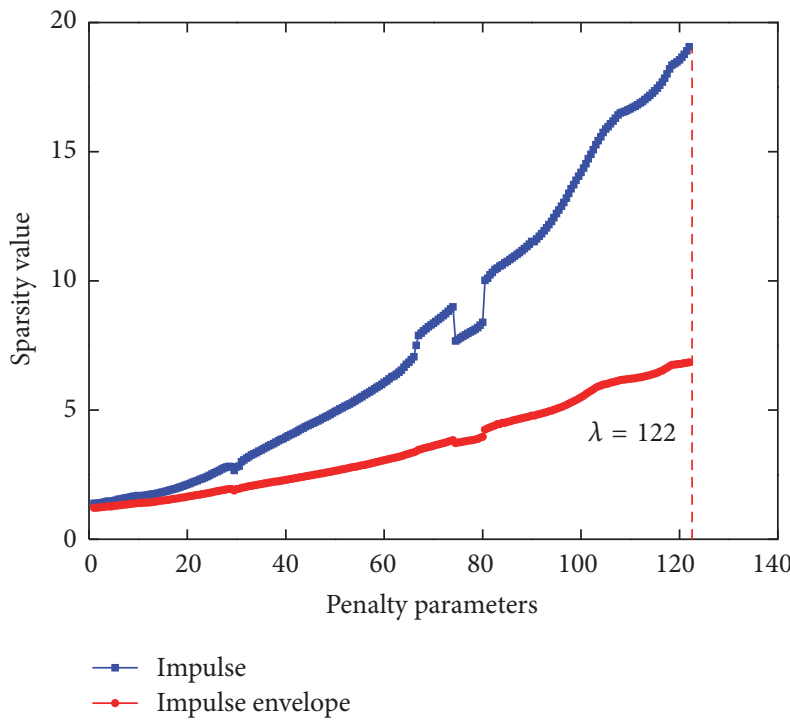

(b)

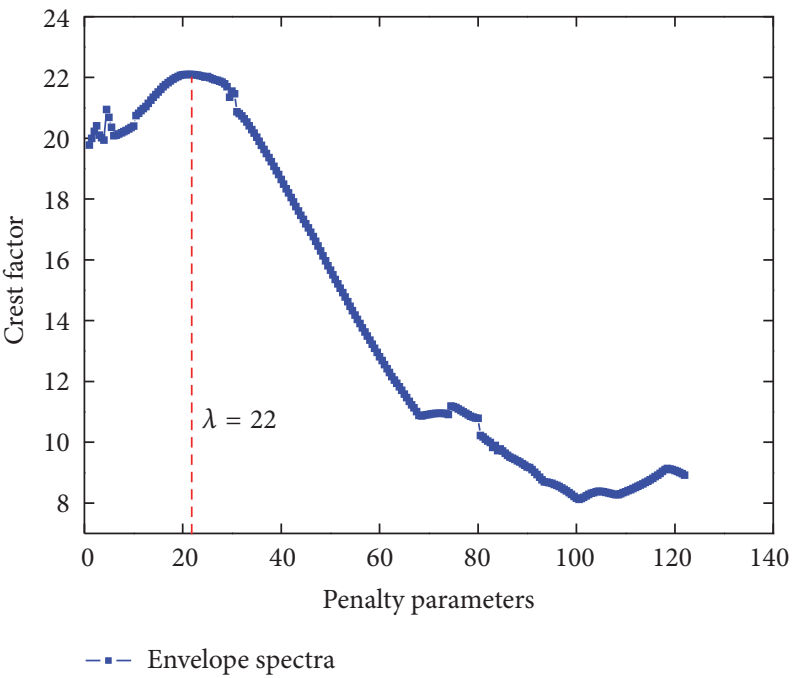

(d)

FIGURE 12: Four indicators for selecting impulses.

Step 1. The signals to be analyzed $\mathbf{a}=\left[\mathbf{a}_{1}{ }^{T}, \ldots, \mathbf{a}_{K}{ }^{T}\right]^{T} \in \mathbb{R}^{n K}$ and resulting interval signals $\mathbf{a}_{k}$ are given.

Step 2. Estimate the number of the atom types based on the dominant frequency analysis.

Step 3. Extract the impulses with the different sparse characteristic through CSR with the different penalty parameters.

Step 4. Select the appropriately sparse impulses for fault judgment.

\section{Simulation Validation}

To illustrate the effectiveness of the proposed method, the acceleration responses $\mathbf{a}(t)$ induced by a bearing fault are simulated by the impulse function of a mass-spring-damper system [5] and expressed as

$$
\mathbf{a}(t)=\sum_{l=1}^{L} A_{l} e^{-\beta\left(t-l T_{p}\right)} \cos \left[\omega\left(t-l T_{p}\right)\right] u\left(t-m l T_{p}\right),
$$

where $A_{l}$ denotes the amplitude of the $l$ th fault impulse and is set as 0.000000005 in this paper, $T_{p}$ denotes the reciprocal of fault-character frequency $f_{p}$ and is set as $49.1 \mathrm{~Hz}$ in this paper, $\beta$ means structural damping coefficient and is set as $1200 \mathrm{Ns} / \mathrm{m}$, $\omega$ denotes nature frequency and is equal to $2000 \mathrm{~Hz}$, and $L$ means the number of the simulated impulses and is equal to 39. The fault simulation signals at a $-7 \mathrm{~dB}$ SNR shown in Figure 3 are generated by mixing the fault response in (11) with white noises. 


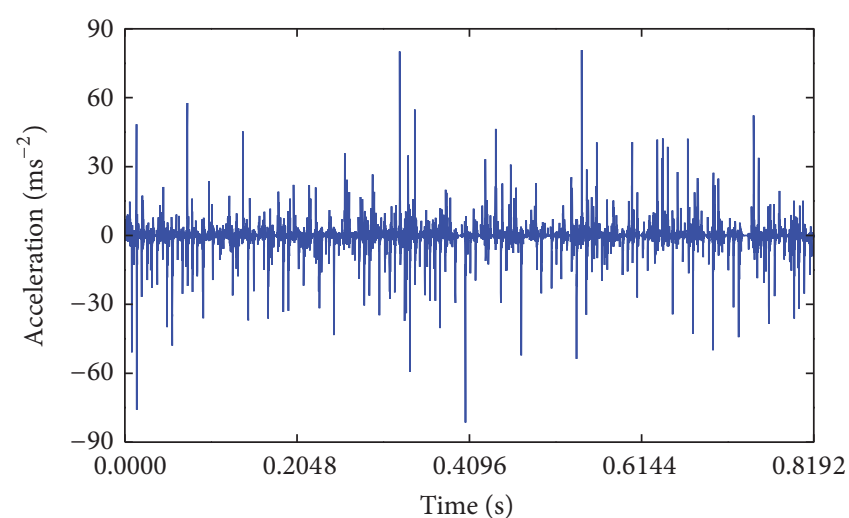

(a)

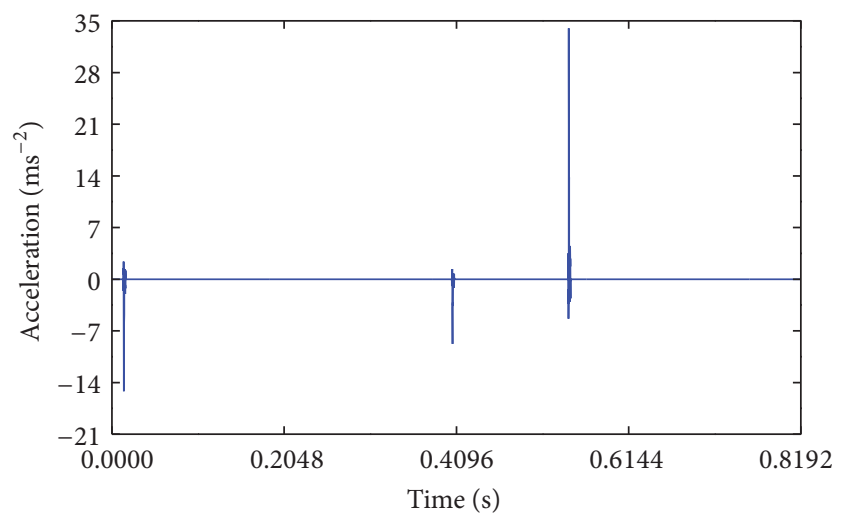

(c)

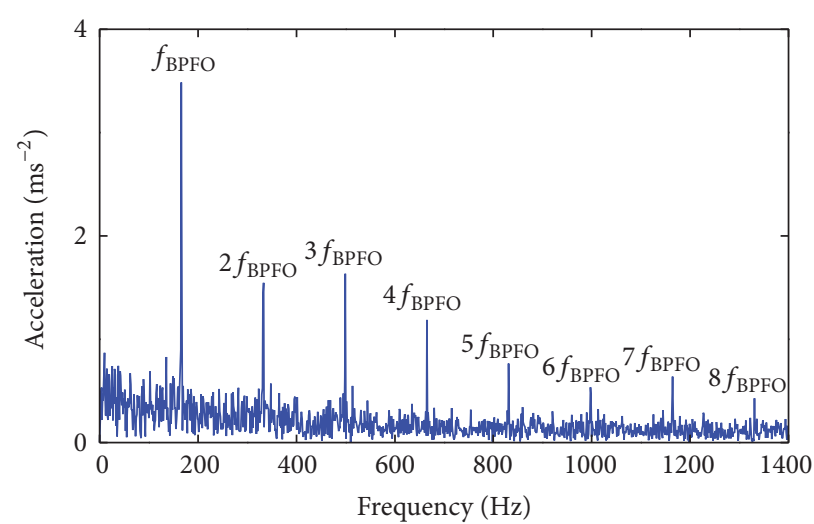

(b)

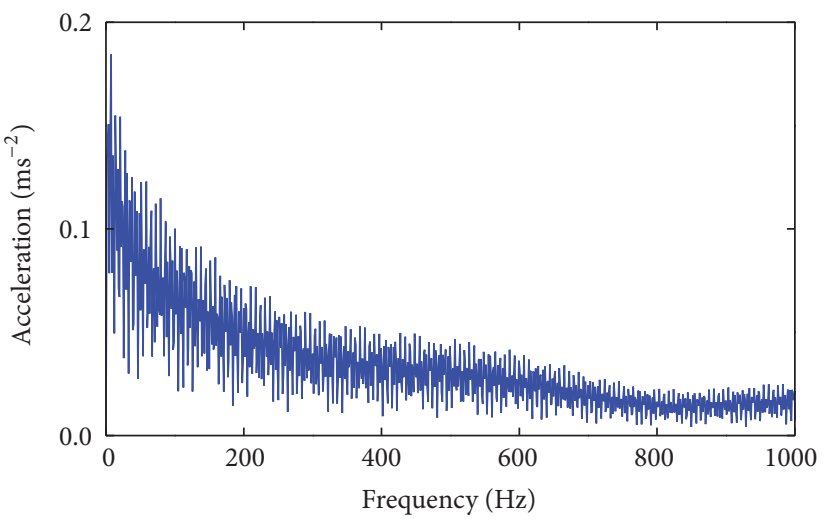

(d)

FIGURE 13: The extracted impulse: (a) by proposed appropriately sparse impulse extraction; (b) the envelope spectra of (a); (c) three other indicators; (d) the envelope spectra of (c).

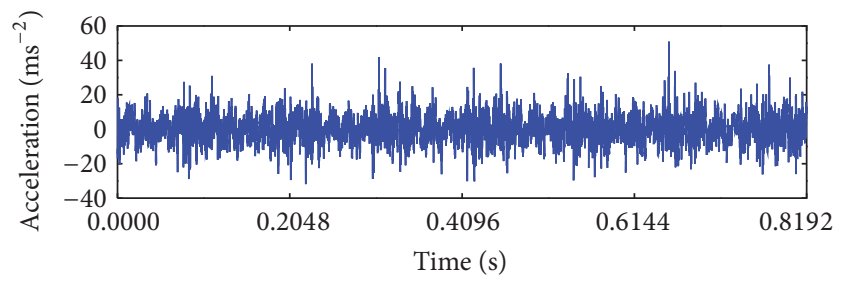

FIGURE 14: Vibration signals of the roller fault.

The impulses with different sparse characteristic are extracted from the signals in Figure 3(b) and shown in Figure 4 . When the penalty parameter increases, the impulses become increasingly sparse. In the range from penalty parameter 1 to penalty parameter 4 , there are false impulses or noise between the true simulation impulses. In the scope of both the penalty parameter 5 and penalty parameter 8 , a part of true impulses are discarded. The impulses with the penalty parameter 4.5 are almost identical to the true impulses of simulation signal in Figure 3(a); there are 39 impulses. The proposed maximal crest factor of envelope spectra is used to select the appropriately sparse impulse. For comparison, the indicators (such as kurtosis value, sparsity value, and permutation entropy value) are computed. These four indicators are shown in Figure 5. Based on the maximal kurtosis value, maximal sparsity value, and minimal permutation entropy value of impulse and impulse envelope, the appropriately sparse impulse should be the impulses with penalty parameter 8 . Based on the proposed maximal crest factor of impulse envelope spectra, the appropriately sparse impulse should be the impulses with penalty parameter 4.5 . This is identical to the previous analysis in Figure 4. The extracted impulses are shown in Figure 6 . The proposed appropriately sparse impulse extraction based on CSR and the maximal crest factor can completely extract 39 impulses embedded in signals in Figure 3(b) and capture the fault-characteristic frequency $(49.1 \mathrm{~Hz})$ and 40-order harmonics. The selected impulses based on three indicators can extract more sparse impulses and capture the 22-order harmonics. The amplitude of the envelope spectra obtained by proposed appropriately sparse impulse method is larger than the selected impulses based on three indicators. These results show that the proposed appropriately sparse impulse method has excellent performance of extracting impulses.

In order to make a comparison with the classical filteringbased demodulation technique, the signals filtered from 


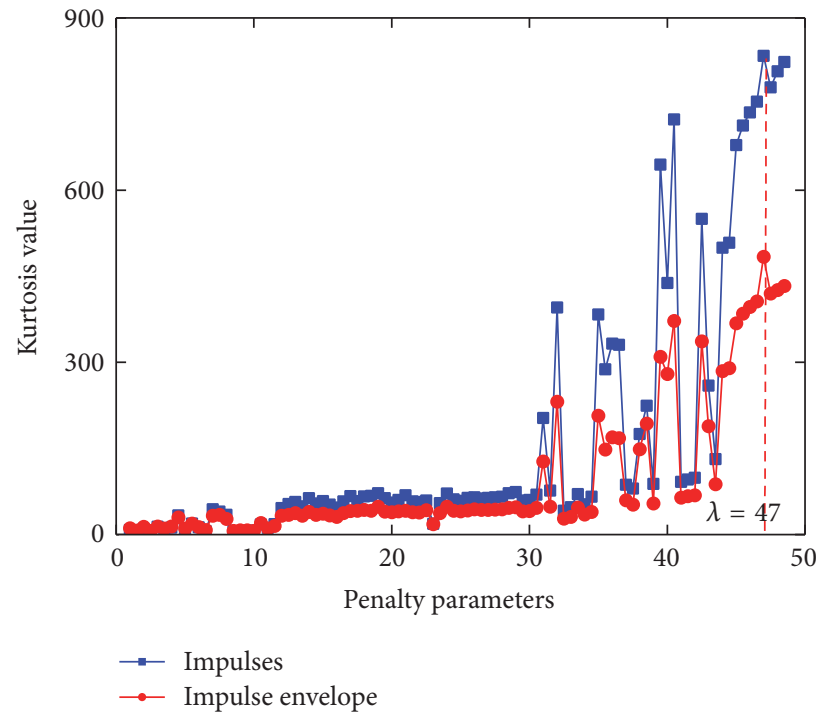

(a)

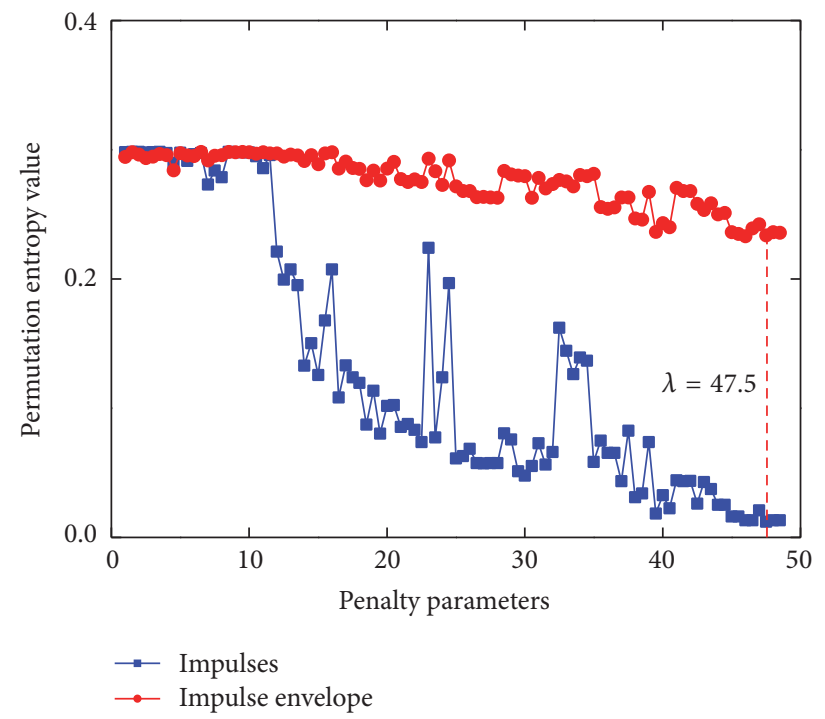

(c)

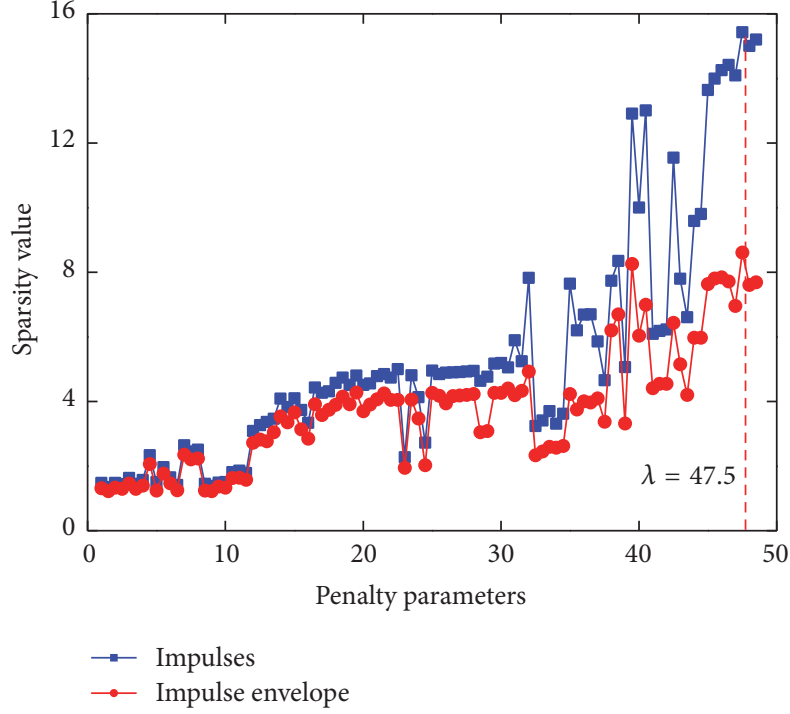

(b)

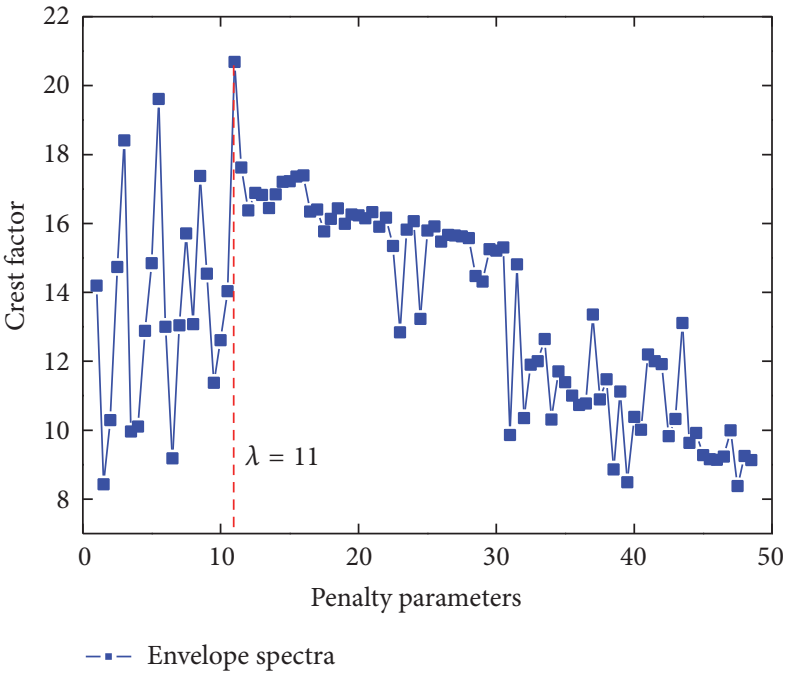

(d)

FIGURE 15: Four indicators for selecting impulses.

simulation signals in Figure 3(a) by a band-pass filter whose centric frequency and bandwidth are $2000 \mathrm{~Hz}$ and $400 \mathrm{~Hz}$, respectively, are shown in Figure 7(a). Hilbert envelope spectra of the filtered signals are shown in Figure 7(b). Compared to Figure 6(a), so many noises exist between the true impulses in Figure $7(\mathrm{a})$ that the 39 impulses embedded in Figure 3(b) cannot be highlighted distinctly. The four harmonics of fault-characteristic frequency are obtained, and much less than 40 harmonics are got by the proposed appropriately sparse impulse extraction. These results show that the proposed appropriately sparse impulse extraction outperforms the classical filtering-based demodulation technique on extracting the impulses. In addition, it is difficult to determine a reasonable resonance band of the real analyzed signals.

\section{Experiment Verifications}

The wheelset bearing test bench used for a practical test of the proposed method is shown in Figure 8. The test bench consists of a motor, driving wheel, loading device, wheelset, and axle box. The motor delivers driving power with different motor speeds. The driving power is conveyed to the driving wheel though rubber belts. The traction power of the driving wheel is then transmitted to the wheelset. The artificial faults on outer race and a roller, shown in Figure 9, are introduced to the roller bearing which is installed in the axle box in Figure 10 on which the accelerometer is mounted. The parameters of the fault bearing are listed in Table 1.

4.1. The Fault Detection on Outer Race. The vibration signals measured from the wheelset bearing system test bench are 


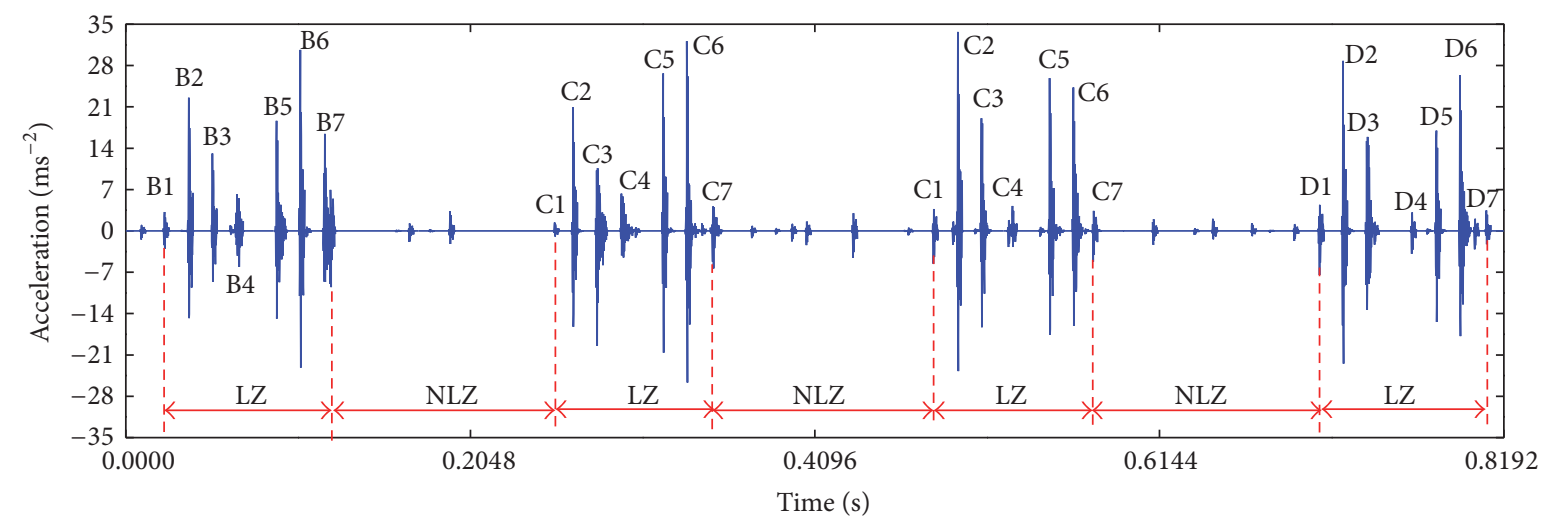

(a)

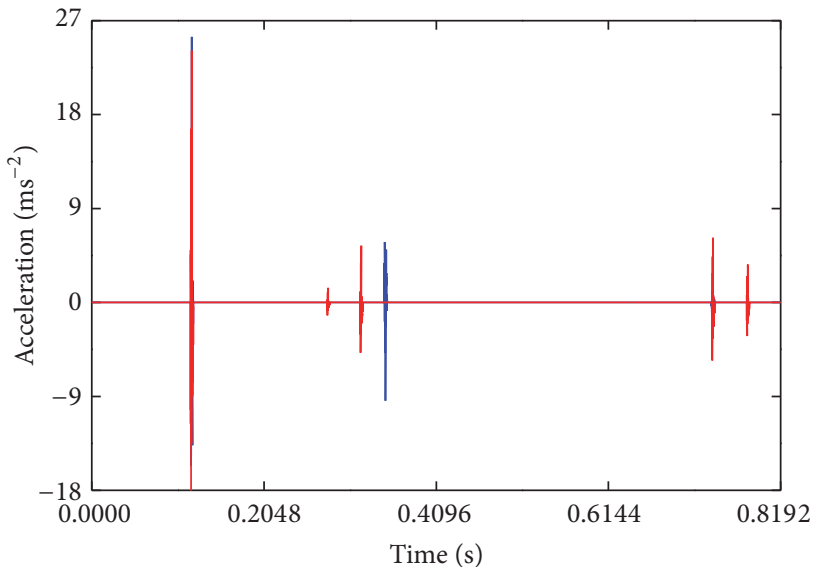

$-\lambda=47$

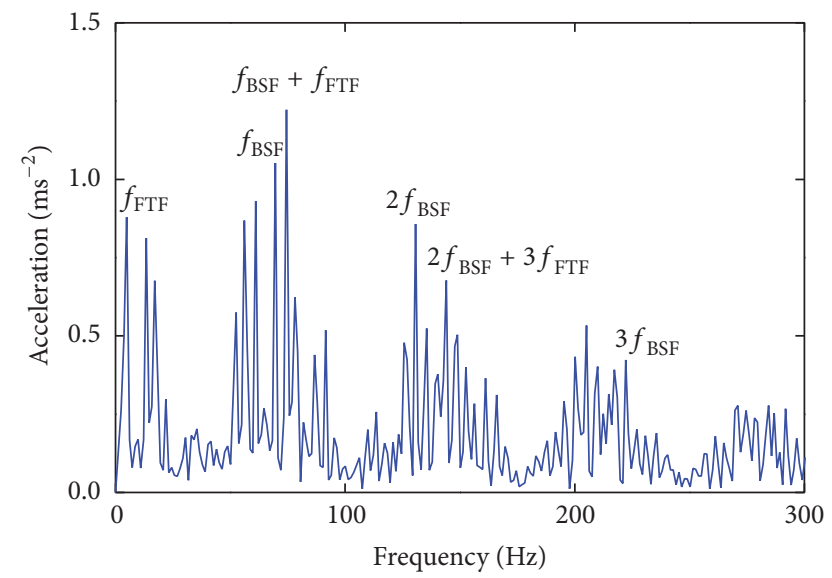

(c)

FIGURE 16: The extracted impulse: (a) by proposed appropriately sparse impulse extraction; (b) three other indicators; (c) the envelope spectra of (a).

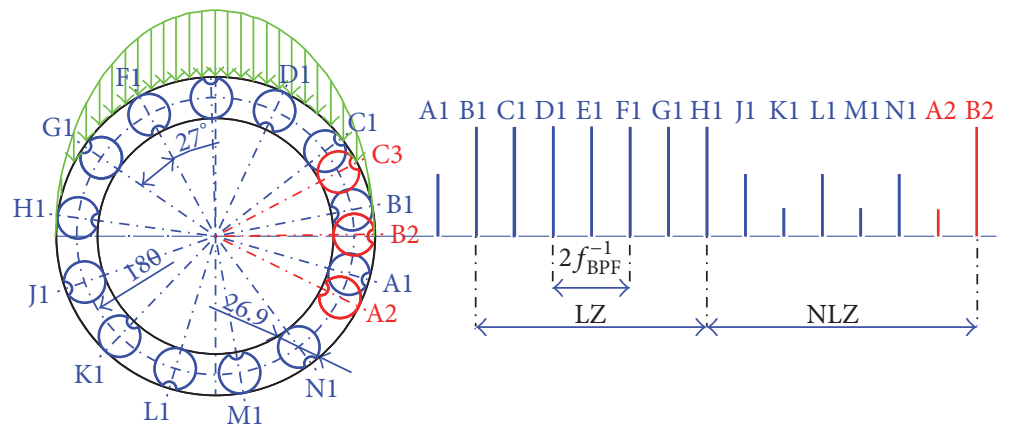

FIGURE 17: Interaction relations between the roller defect and inner-outer races.

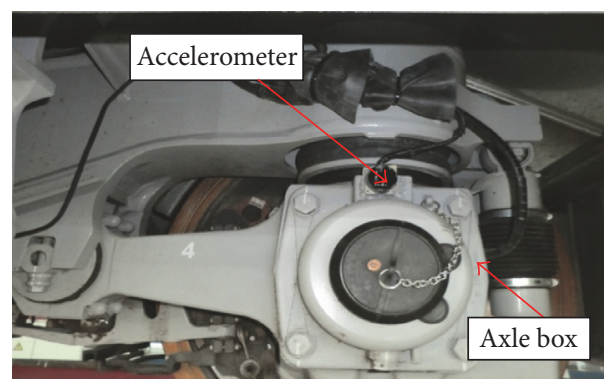

FIGURE 18: Measurement sensor installed on axle box in a high-speed train. 


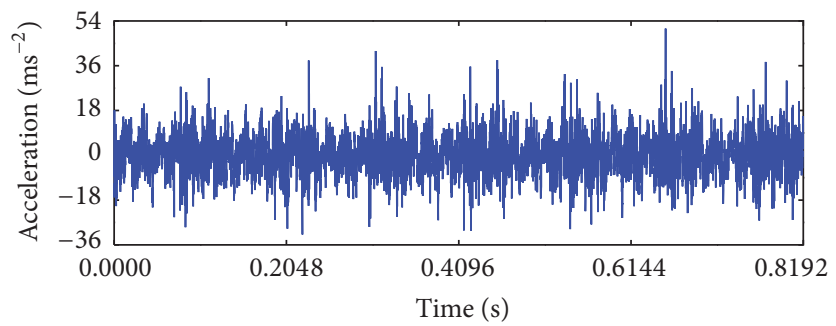

FIGURE 19: The vibration signal measured from the axle box of running high-speed train.

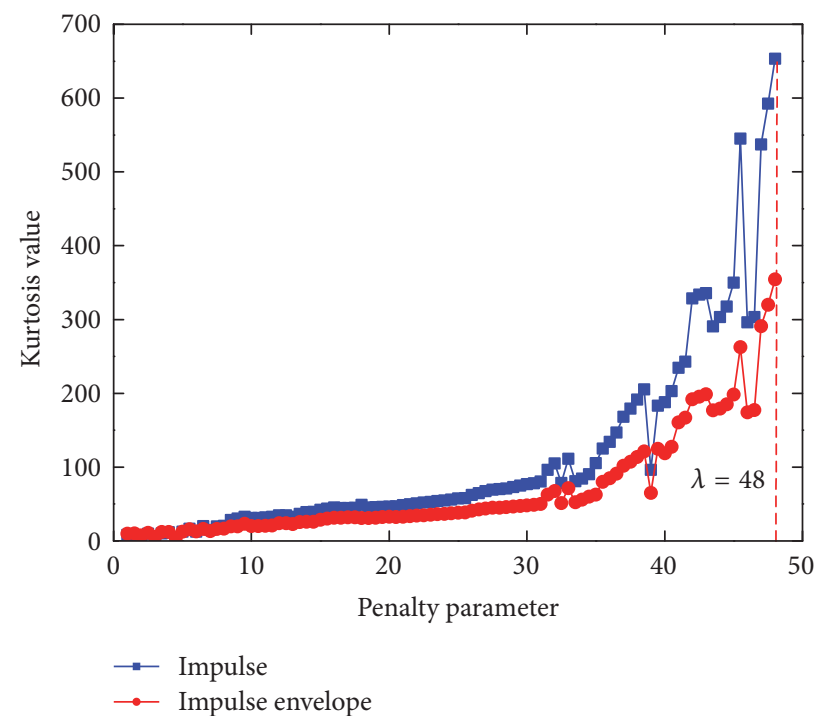

(a)

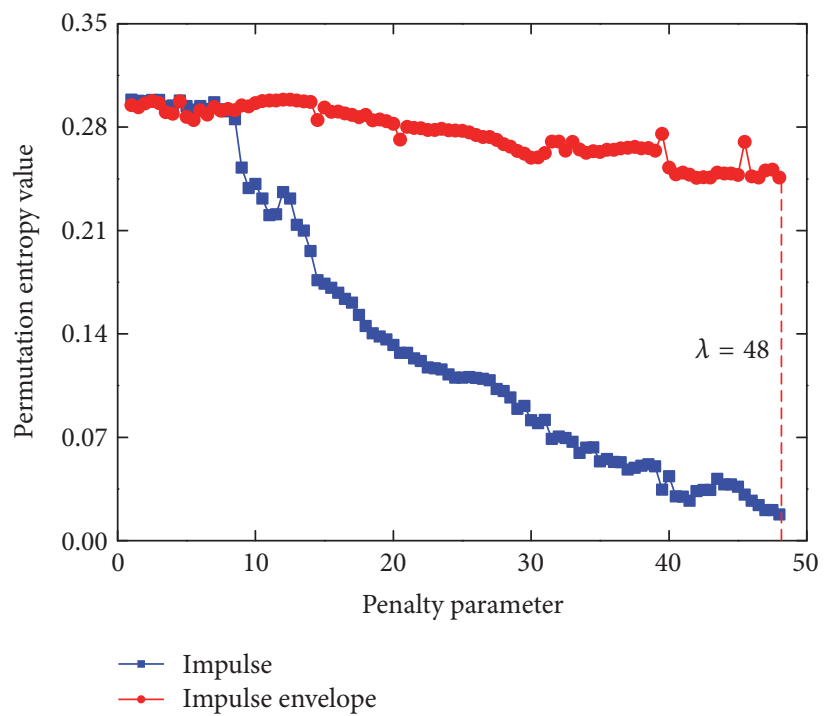

(c)

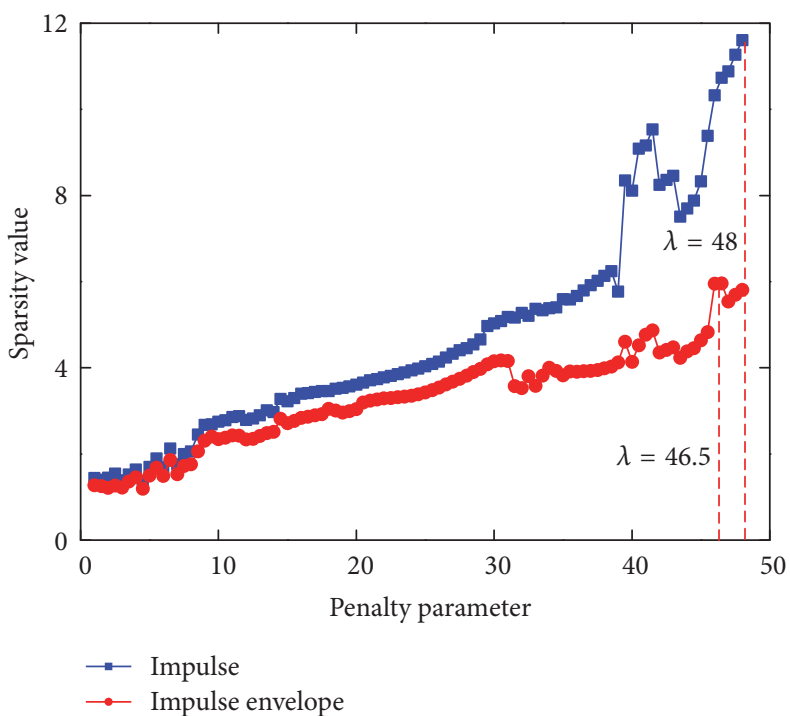

(b)

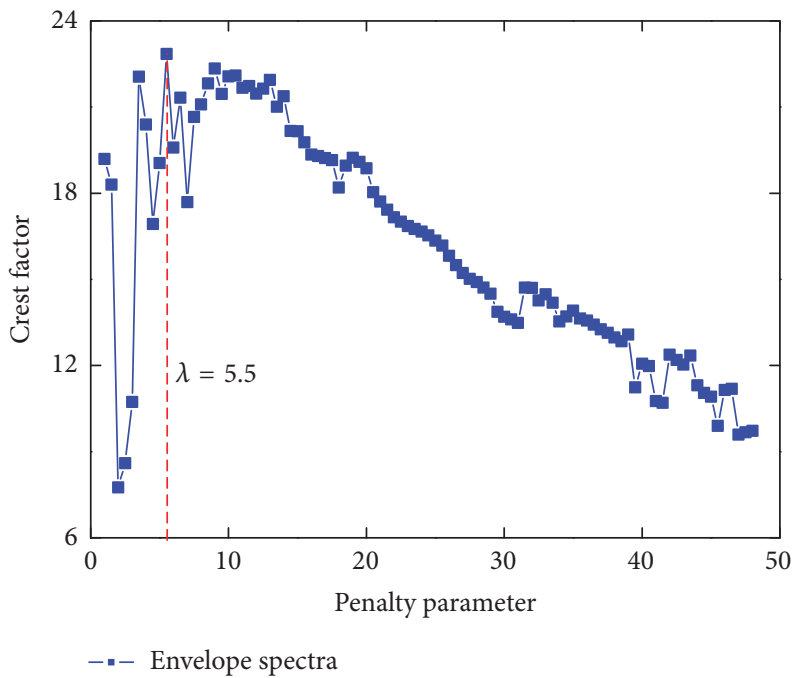

(d)

FIGURE 20: Four indicators for selecting impulses.

TABLE 1: The parameters of the test rolling bearing. 


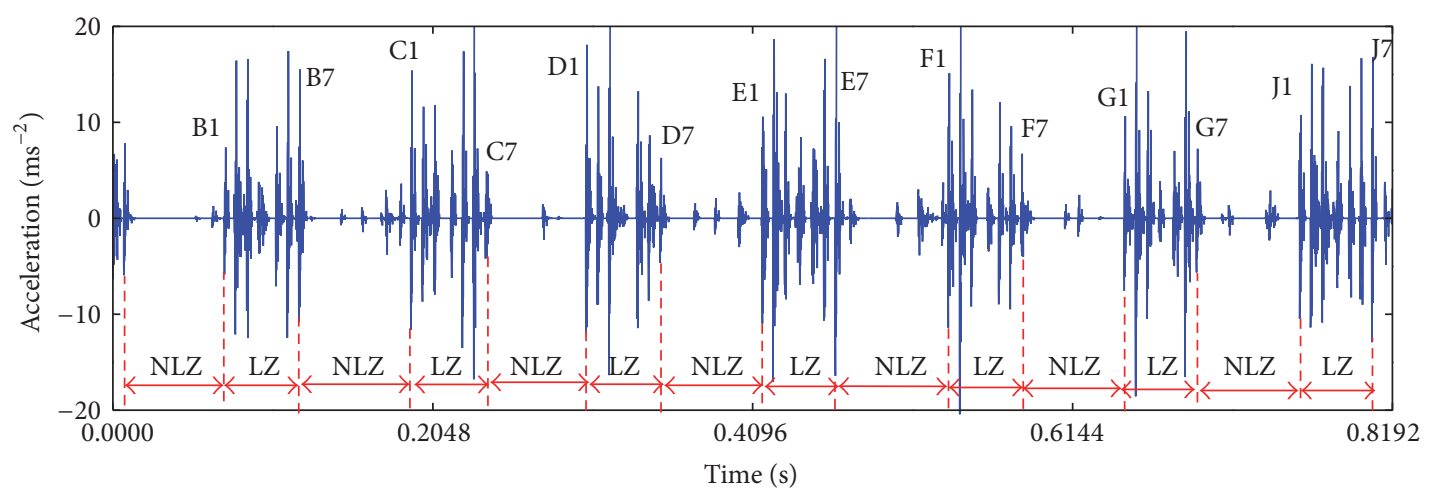

(a)

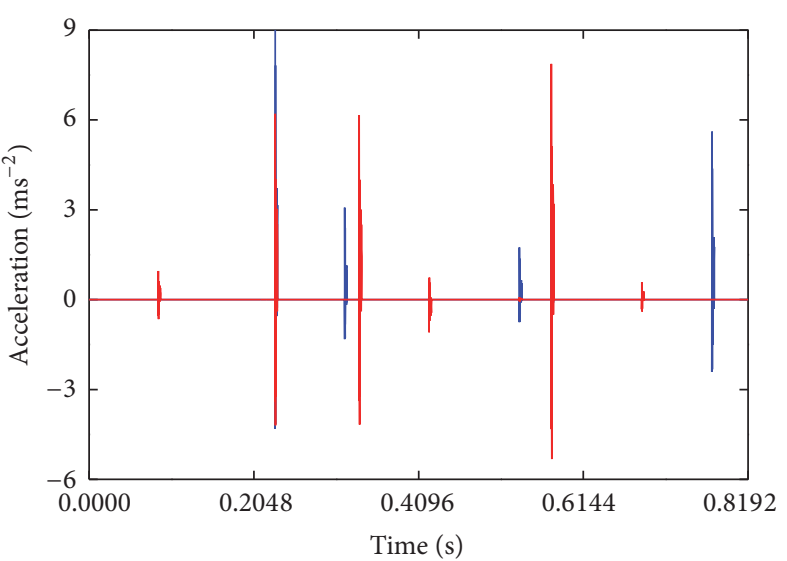

$\lambda=46.5$

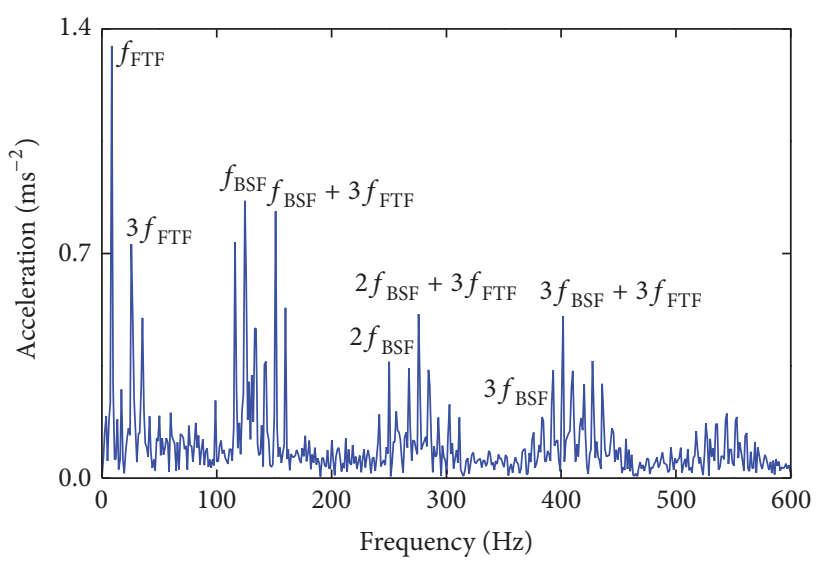

(b)

(c)

Figure 21: The extracted impulse related to atom 1: (a) by proposed appropriately sparse impulse extraction; (b) three other indicators; (c) the envelope spectra of (a).

shown in Figure 11 when the outer race of the wheelset bearing has the fault in Figure 9(a).

The 122 impulses series extracted from the vibration signals in Figure 11 by CSR with different penalty parameters cannot be displayed due to large number (similar to Figure 4). The kurtosis value, sparsity value, permutation entropy value, and crest factor of these 122 impulses series are calculated and shown in Figure 12. So, the impulses with penalty parameters 20 and 122 are selected to judge fault. The impulse with penalty parameters 22 is the one extracted by the proposed appropriately sparse impulse method shown in Figure 13(a). Figure 13(b) shows the outer-race fault-characteristic frequency $f_{\mathrm{BPFO}}$ and its 8 -order harmonics. The outer-race faultcharacteristic frequency $f_{\mathrm{BPFO}}$ is expressed as

$$
f_{\mathrm{BPFO}}=\frac{N_{b}}{2}\left(1-\frac{B_{d}}{P_{d}} \cos (\phi)\right) f_{w}
$$

where $N_{b}$ denotes roller number, $B_{d}$ denotes roller diameter, $P_{d}$ means pitch diameter, $\phi$ means contact angle, and $f_{w}$ denotes the rotation speed.

The impulses with penalty parameter 122 are the impulses selected by three other indicators and shown in Figure 13(c),
Figure 13(d) shows the Hilbert envelope spectra of Figure 13(c), and they fail to capture the useful feature information for fault detection.

4.2. The Fault Detection on Roller. The collected vibration signals from the wheelset bearing system test bench are shown in Figure 14, when the roller of the wheelset bearing has the fault shown in Figure 9(b).

The kurtosis value, sparsity value, permutation entropy value, and crest factor of the extracted 96 impulse series with different sparse characteristic are computed and shown in Figure 15. The three impulses series with penalty parameters $47,47.5$, and 11 are selected. The impulses with penalty parameter 11 are the ones extracted by the proposed appropriately sparse impulse extraction and shown in Figure 16(a). In order to understand the vibrational phenomenon of the extracted impulses in Figure 16(a), the interaction relations of the roller defect and its matching races are described and illustrated in Figure 17. When a defective roller leaves the no-load zone (NLZ) of the wheelset bearing from position $\mathrm{Al}$ and enters the load zone (LZ) of the wheelset bearing at location B1 after a half-rotation of the roller, the interactions between the roller defect and inner race generate impulse Bl. After 


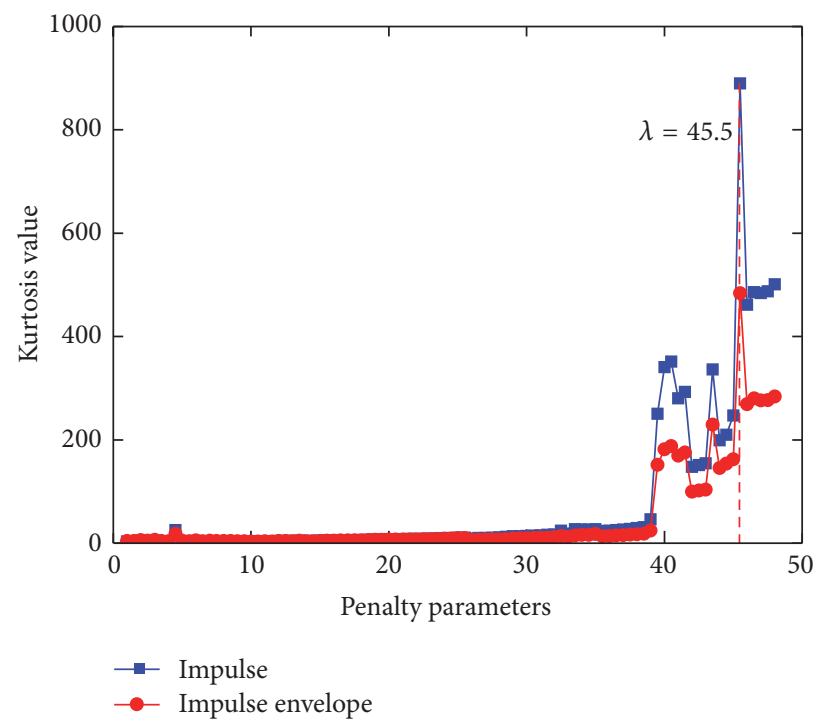

(a)

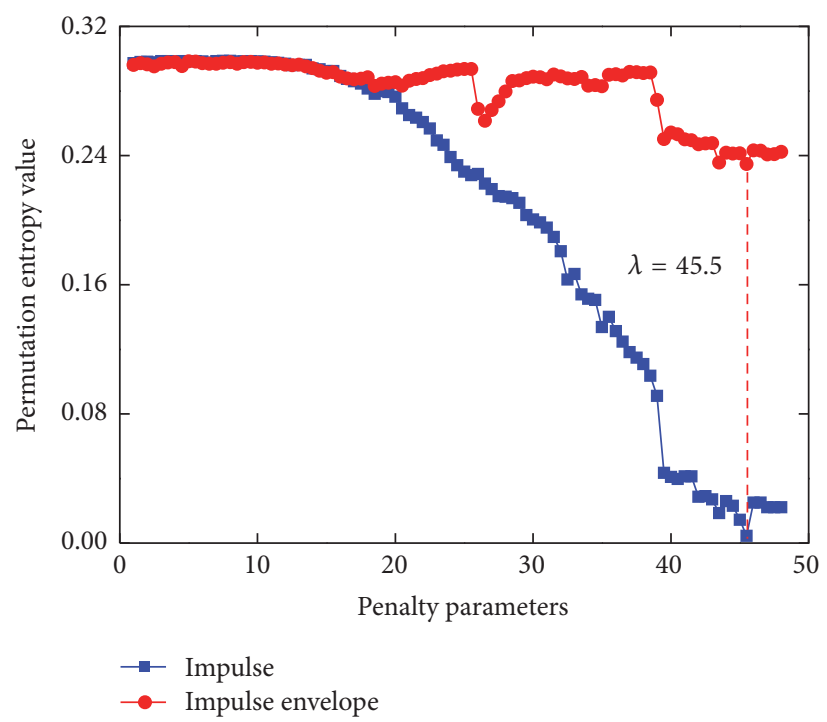

(c)

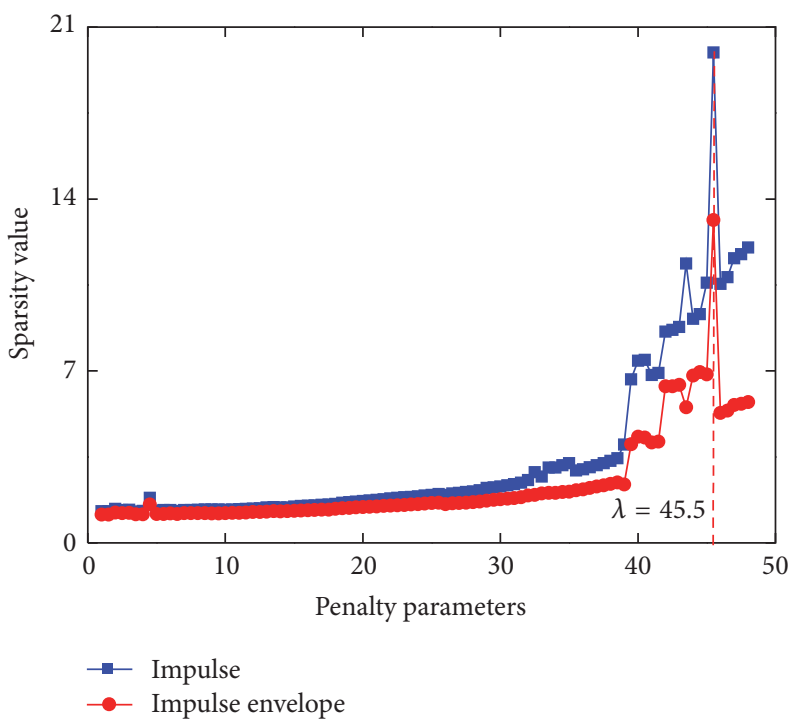

(b)

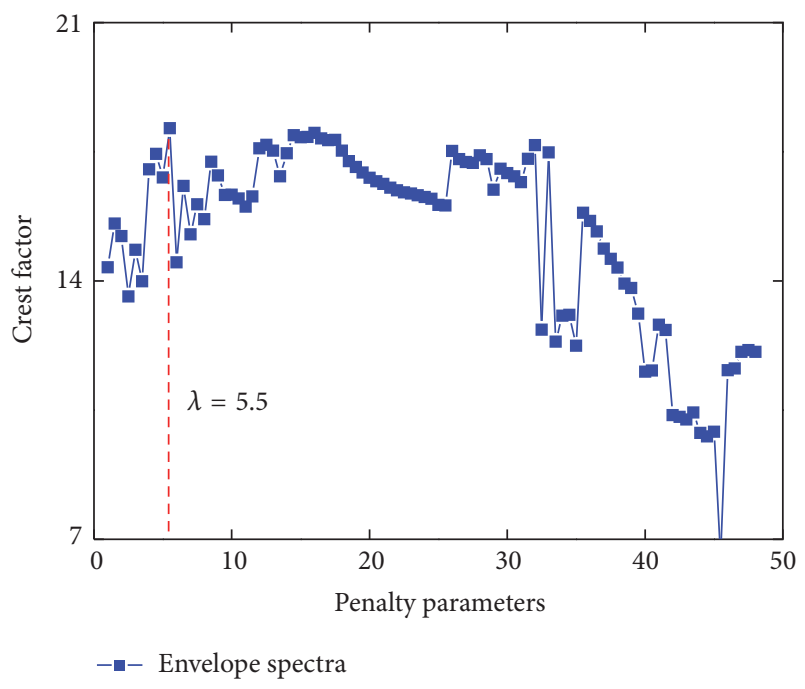

(d)

FIGURE 22: Four indicators for selecting impulses.

a half-rotation of the roller, the impulse $\mathrm{Cl}$ induced by the interactions between the roller defect and outer race emerges. Similarly, the impulse sequences (e.g., D1, E1, F1, G1, and $\mathrm{H} 1)$ are produced through the alternating interactions of the roller defect with the outer race and inner race. There are 7 impulses in the LZ of wheelset bearing. At position J1, the defective roller enters the NLZ of the bearing, and the dynamic interactions between the roller and races are much smaller than those in the LZ of the bearing because the forces applied on the roller only include small self-gravity and centrifugal forces when the defective roller lies in the NLZ of the bearing. After passing over the entire NLZ (K1, L1, M1, N1, and A2), the defective roller enters the LZ. When the defective roller alternatively enters and leaves the LZ of wheelset bearing, the position and direction of the forces excited by the defect applied on races and roller vary. As a result, the amplitudes of the impulses induced by roller defect are modulated by the cage rotation frequency $f_{\mathrm{FTF}}$. The time interval between both the adjacent impulses at a constant rotational speed is properly equal to the roller faultcharacteristic frequency $f_{\mathrm{BSF}}$ which is expressed as

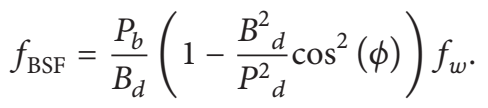

In Figure 16(a), there are properly 7 impulses (e.g., from impulse B1 to impulse B7) in the LZ of wheelset bearing, and there are almost impulses in NLZ of the wheelset bearing due to the small forces applied on defective roller. These show that the proposed appropriately sparse impulse extraction can accurately characterize the dynamic behaviour of defective roller; Figure 16(b) shows the Hilbert envelope spectra of Figure 16(a) that capture the roller fault-characteristic frequency 


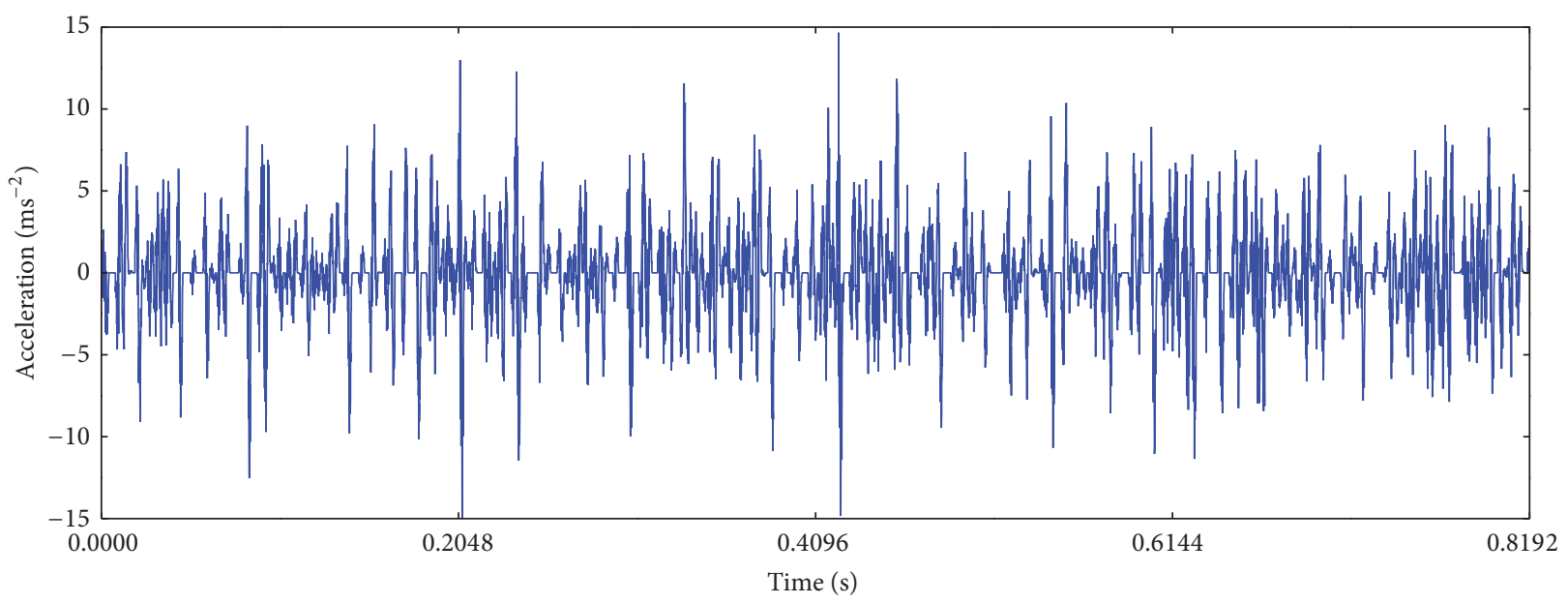

(a)

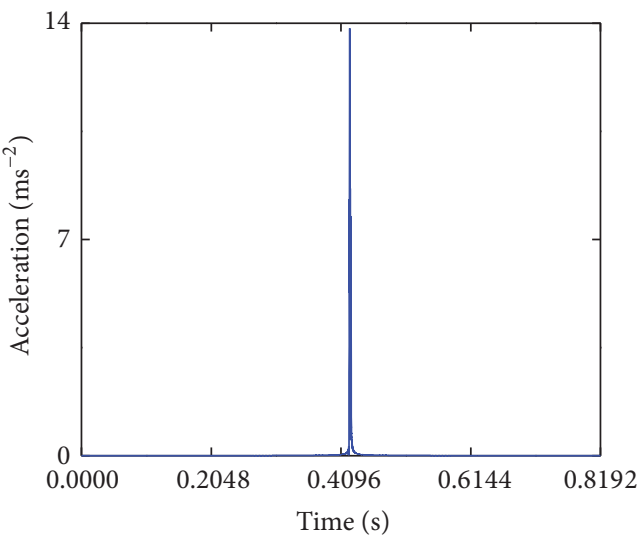

(b)

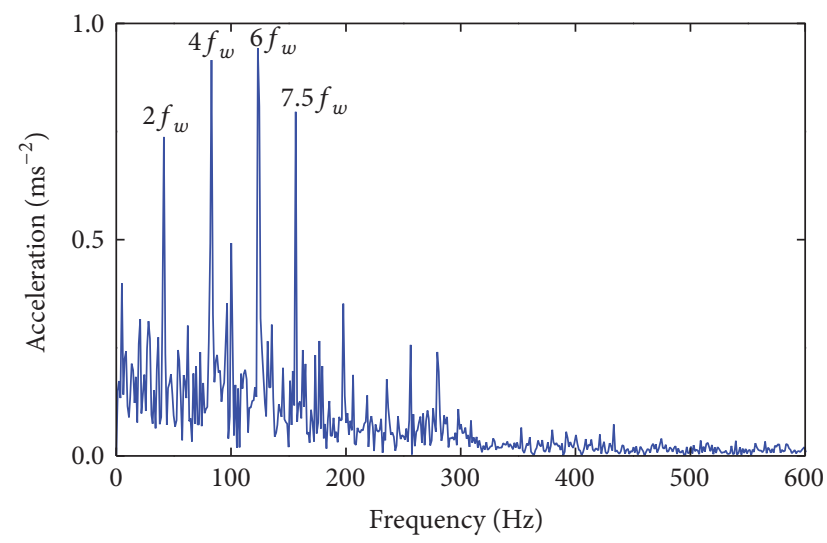

(c)

FIGURE 23: The extracted impulse related to atom 2: (a) by proposed appropriately sparse impulse extraction; (b) three other indicators; (c) the envelope spectra of (a).

and its 3-order harmonics for fault judgment. Meanwhile, the phenomenon that the amplitudes of the impulses induced by roller fault are modulated by cage rotation frequency $f_{\mathrm{FTF}}$ is observed in Figures 16(a) and 16(c). The impulses selected by other three indicators shown in Figure 16(b) fail to capture any useful information for fault judgments.

\section{Practical Application}

To test the fault detection performance of the proposed method during the practical running condition of high-speed train, an accelerometer is installed on axle box of high-speed train and shown in Figure 18. The vibration acceleration signals are collected from the axle box of running high-speed train at the speed of $200 \mathrm{kmh}^{-1}$ when the roller of wheelset bearing has a defect and shown in Figure 19.

The two types of atoms are embedded in vibration signals in Figure 19 through estimating the number of the atom types. Aiming at atom 1, the kurtosis value, sparsity value, permutation entropy value, and crest factor of the extracted 96 impulse series with different sparsity characteristic are computed and shown in Figure 20. The impulse series with penalties $48,46.5$, and 5.5 are selected and shown in Figure 21. The impulses with penalty 5.5 in Figure 21(a) are the ones extracted by the proposed appropriately sparse impulse extraction. There are 7 impulses in the $\mathrm{LZ}$ of wheelset bearing (e.g., from impulse B1 to impulse B7); when the defective roller enters the NLZ of wheelset bearing, there are almost significant impulses with a large shock amplitude compared to the LZ. This is identical to the conclusion drawn by the analysis of the interaction relations between the roller defect and inner-outer races. The envelope spectra of Figure 21(a) are shown in Figure 21(c) and can well capture the roller fault-characteristic frequency $f_{\mathrm{BSF}}$ and its 3-order harmonics for fault judgment. The number of the impulses selected by other three measure indicators in Figure 21(b) obviously is less than the true one induced by roller faults, so that these impulses cannot provide enough information about judging the roller fault. These illustrate that the proposed appropriately sparse impulse extraction can effectively capture the impulses induced by roller faults. Aiming at atom 2, the kurtosis value, sparsity value, permutation entropy value, and crest factor of the extracted 96 impulse series with different sparsity characteristic are computed and shown in Figure 22. 
The impulse series with penalties 45.5 and 5.5 are selected and shown in Figure 23. The impulse series with penalty parameter 5.5 are the ones obtained by the proposed appropriately sparse impulse extraction in Figure 23(a); its envelope spectra shown in Figure 23(c) capture 2, 4, 6, and 7.5 times the wheelset rotational frequency $f_{w}$. This illustrates that the wheel tread is out of roundness. The impulse series in Figure 23(b) are selected by other three indicators; only one shock exists in the impulse series. So the proposed appropriately sparse impulse extraction makes full use of the capability of CSR of extracting impulses embedded vibration signals and the capability of crest factor of determining the sparsity of impulses induced by the roller faults and unevenness of wheel tread.

\section{Conclusion}

In this paper, a novel fault detection method, the appropriately sparse impulse extraction, is proposed based on the combination of CSR, ENA, and crest factor.

(1) CSR provides the excellent framework of extracting the impulses induced by the bearing faults and wheelrail interactions with the virtues of sparsity, flexibility, and superresolution. ENA can estimate the number of atoms embedded in vibration signals. Crest factor can select the impulse series with the most reasonable sparsity characteristic which makes the number of extracted impulses almost equal to the true one of impulses induced by bearing faults.

(2) The appropriately sparse impulse extraction not only can well capture the fault-characteristic frequencies and their harmonics for fault judgments but also analyzes the dynamic behaviour process of defective elements and their matching surfaces.

(3) The appropriately sparse impulse extraction can isolate the impulses induced by the bearing faults and wheel tread unevenness. It is every suitable for detecting fault of wheelset bearing.

In this paper, only one impulse series is selected and used to judge faults based on the maximal crest factor. The combined application of multi-impulse series with different penalty parameters should be further studied.

\section{Conflicts of Interest}

The authors declare that they have no conflicts of interest.

\section{Acknowledgments}

This work is supported by the National Natural Science Foundation of China (nos. 51305358, 51375405, and 51475387) and the Research Fund of State Key Laboratory of Traction Power (no. 2015TPL_T17).

\section{References}

[1] H. C. Chose, Y. Wan, and A. K. Chan, "Neural pattern identification of railroad wheel-bearing faults from audible acoustic signals: comparison of FFT, CWT, and DWT features," in Proceedings of the SPIE-The International Society for Optical Engineering, pp. 480-496, 1997.

[2] J. Donelson III. and R. L. Dicus, "Bearing defect detection using on-board accelerometer measurements," in Proceedings of the ASME/IEEE 2002 Joint Rail Conference, RTD 2002, pp. 95-102, Washington, usa, April 2002.

[3] H. R. Cao, F. Fan, K. Zhou, and Z. J. He, "Wheel-bearing fault diagnosis of trains using empirical wavelet transform," Measurement, vol. 82, pp. 439-449, 2016.

[4] H. Ocak, K. A. Loparo, and Discenzo F. M., "Online tracking of bearing wear using wavelet packet decomposition and probabilistic modelling-A method for bearing prognostics," Journal of Sound and Vibration, vol. 302, pp. 951-961, 2007.

[5] Y. G. Lei, Z. J. He, and Y. Zi, "New clustering algorithmbased fault diagnosis using compensation distance evaluation techniquetechnique," Mechanical Systems and Signal Processing, vol. 22, pp. 419-435, 2008.

[6] G. He, K. Ding, and H. Lin, "Fault feature extraction of rolling element bearings using sparse representation," Journal of Sound and Vibration, vol. 366, pp. 514-527, 2016.

[7] R. Golafshan and K. Y. Sanliturk, "SVD and hankel matrix based de-noising approach for ball bearing fault detection and its assessment using artificial faults," Mechanical Systems and Signal Processing, vol. 70, no. 71, pp. 36-50, 2016.

[8] D. Wang, "An extension of the infograms to novel Bayesian inference for bearing fault feature identification," Mechanical Systems and Signal Processing, vol. 80, pp. 19-30, 2016.

[9] D. Wang and K. Tsui, "Dynamic Bayesian wavelet transform: New methodology for extraction of repetitive transients," Mechanical Systems and Signal Processing, vol. 88, pp. 137-144, 2017.

[10] H. Jiang, C. Li, and H. Li, "An improved EEMD with multiwavelet packet for rotating machinery multi-fault diagnosis," Mechanical Systems and Signal Processing, vol. 36, no. 2, pp. 225239, 2013.

[11] X. F. Chen, Z. H. Du, J. Li, X. Li, and H. Zhang, "Compressed sensing based on dictionary learning for extracting impulse components," Signal Processing, vol. 96, pp. 94-109, 2014.

[12] R. Jiang, H. Qiao, and B. Zhang, "Efficient fisher discrimination dictionary learning," Signal Processing, vol. 128, pp. 28-39, 2016.

[13] M. Aharon, M. Elad, and A. Bruckstein, "K-SVD: an algorithm for designing overcomplete dictionaries for sparse representation," IEEE Transactions on Signal Processing, vol. 54, no. 11, pp. 4311-4322, 2006.

[14] G. Davis, S. Mallat, and M. Avellaneda, "Adaptive greedy approximations," Constructive Approximation, vol. 13, no. 1, pp. 57-98, 1997.

[15] A. M. Tillmann, "On the computational intractability of exact and approximate dictionary learning," IEEE Signal Processing Letters, vol. 22, pp. 45-49, 2015.

[16] Y. C. Pati, R. Rezaiifar, and P. S. Krishnaprasad, "Orthogonal matching pursuit: recursive function approximation with applications to wavelet decomposition," in Proceedings of the 27th Annu. Asilomar Conf. Signals, Systems, and Computers, Pacific Grove, CA, vol. 1, pp. 40-44, Pacific Grove, CA, 1993.

[17] J. A. Tropp, "Greed is good: algorithmic results for sparse approximation," Institute of Electrical and Electronics Engineers. 
Transactions on Information Theory, vol. 50, no. 10, pp. 22312242, 2004.

[18] T. T. Cai and L. Wang, "Orthogonal matching pursuit for sparse signal recovery with noise," IEEE Transactions on Information Theory, vol. 57, no. 7, pp. 4680-4688, 2011.

[19] A. Huang, G. Guan, and Q. Wan, "A block orthogonal matching pursuit algorithm based on sensing dictionary," International Journal of Physical Sciences, vol. 6, pp. 992-999, 2011.

[20] D. Needell and J. A. Troppb, "CoSaMP: iterative signal recovery from incomplete and inaccurate samples," Applied and Computational Harmonic Analysis, vol. 26, pp. 301-321, 2009.

[21] W. Dai and O. Milenkovic, "Subspace pursuit for compressive sensing signal reconstruction," IEEE Transactions on Information Theory, vol. 55, no. 5, pp. 2230-2249, 2009.

[22] M. A. T. Figueiredo, R. D. Nowak, and S. J. Wright, "Gradient projection for sparse reconstruction: application to compressed sensing and other inverse problems," IEEE Journal on Selected Topics in Signal Processing, vol. 1, no. 4, pp. 586-597, 2007.

[23] S. J. Wright, R. D. Nowak, and M. A. T. Figueiredo, "Sparse reconstruction by separable approximation," IEEE Transactions on Signal Processing, vol. 57, no. 7, pp. 2479-2493, 2009.

[24] I. Daubechies, M. Defrise, and C. de Mol, "An iterative thresholding algorithm for linear inverse problems with a sparsity constraint," Communications on Pure and Applied Mathematics, pp. 1413-1457, 2004.

[25] A. Beck and M. Teboulle, "A fast iterative shrinkagethresholding algorithm for linear inverse problems," SIAM Journal on Imaging Sciences, vol. 2, pp. 183-202, 2009.

[26] S. Yun and K.-C. Toh, "A coordinate gradient descent method for $l_{1}$-regularized convex minimization," Computational Optimization and Applications, vol. 48, no. 2, pp. 273-307, 2011.

[27] W. Yin, S. Osher, D. Goldfarb, and J. Darbon, "Bregman iterative algorithms for $\ell_{1}$-minimization with applications to compressed sensing," SIAM Journal on Imaging Sciences, vol. 1, no. 1, pp. 143-168, 2008.

[28] D. L. Donoho, "For most large underdetermined systems of linear equations, the minimal $l_{1}$-norm solution is also the sparsest solution," Communications on Pure and Applied Mathematics, vol. 59, no. 7, pp. 907-934, 2006.

[29] S. S. Chen, D. L. Donoho, and M. A. Saunders, "Atomic decomposition by basis pursuit," SIAM Journal on Scientific Computing, vol. 20, no. 1, pp. 33-61, 1998.

[30] Z. Feng and F. Chu, "Application of atomic decomposition to gear damage detection," Journal of Sound and Vibration, vol. 302, no. 1-2, pp. 138-151, 2007.

[31] K. Skretting and K. Engan, "Recursive least squares dictionary learning algorithm," IEEE Transactions on Signal Processing, vol. 58, no. 4, pp. 2121-2130, 2010.

[32] R. Rubinstein, T. Peleg, and M. Elad, "Analysis K-SVD: a dictionary-learning algorithm for the analysis sparse model," IEEE Transactions on Signal Processing, vol. 61, no. 3, pp. 661677, 2013.

[33] J. Mairal, F. Bach, and J. Ponce, "Online dictionary learning for sparse coding," in Proceedings of the 26th Annual International Conference on Machine Learning (ICML '09), pp. 689-696, 2009.

[34] K. Engan, K. Skretting, and J. H. Husøy, "Family of iterative LSbased dictionary learning algorithms, ILS-DLA, for sparse signal representation," Digital Signal Processing: A Review Journal, vol. 17, no. 1, pp. 32-49, 2007.

[35] E. C. Smith and M. S. Lewicki, "Efficient auditory coding," Nature, vol. 439, no. 7079, pp. 978-982, 2006.
[36] T. Blumensath and M. Davies, "Sparse and shift-invariant representations of music," IEEE Transactions on Audio, Speech and Language Processing, vol. 14, no. 1, pp. 50-57, 2006.

[37] C. Rusu, B. Dumitrescu, and S. A. Tsaftaris, "Explicit shiftinvariant dictionary learning," IEEE Signal Processing Letters, vol. 21, no. 1, pp. 6-9, 2014.

[38] L. Cui, N. Wu, C. Ma, and H. Wang, "Quantitative fault analysis of roller bearings based on a novel matching pursuit method with a new step-impulse dictionary," Mechanical Systems and Signal Processing, vol. 68-69, pp. 34-43, 2016.

[39] H. Yang, J. Mathew, and L. Ma, "Fault diagnosis of rolling element bearings using basis pursuit," Mechanical Systems and Signal Processing, vol. 19, no. 2, pp. 341-356, 2005.

[40] Y. Qin, Y. Mao, and B. Tang, "Vibration signal component separation by iteratively using basis pursuit and its application in mechanical fault detection," Journal of Sound and Vibration, vol. 332, no. 20, pp. 5217-5235, 2013.

[41] W. Fan, G. Cai, Z. K. Zhu, C. Shen, W. Huang, and L. Shang, "Sparse representation of transients in wavelet basis and its application in gearbox fault feature extraction," Mechanical Systems and Signal Processing, vol. 56-57, pp. 230-245, 2015.

[42] Y. Wang, J. Xiang, Q. Mo, and S. He, "Compressed sparse timefrequency feature representation via compressive sensing and its applications in fault diagnosis," Measurement, vol. 68, pp. 7081, 2015.

[43] H. Tang, J. Chen, and G. Dong, "Sparse representation based latent components analysis for machinery weak fault detection," Mechanical Systems and Signal Processing, vol. 46, no. 2, pp. 373388, 2014.

[44] H. Liu, C. Liu, and Y. Huang, "Adaptive feature extraction using sparse coding for machinery fault diagnosis," Mechanical Systems and Signal Processing, vol. 25, no. 2, pp. 558-574, 2011.

[45] H. Zhou, J. Chen, G. Dong, and R. Wang, "Detection and diagnosis of bearing faults using shift-invariant dictionary learning and hidden Markov model," Mechanical Systems and Signal Processing, vol. 72-73, pp. 65-79, 2016.

[46] Z. Feng and M. Liang, "Complex signal analysis for planetary gearbox fault diagnosis via shift invariant dictionary learning," Measurement, vol. 90, pp. 382-395, 2016.

[47] B. Wohlberg, "Efficient algorithms for convolutional sparse representations," IEEE Transactions on Image Processing, vol. 25, no. 1, pp. 301-315, 2016.

[48] J. Antoni and R. B. Randall, "A stochastic model for simulation and diagnostics of rolling element bearings with localized faults," Journal of Vibration and Acoustics, Transactions of the ASME, vol. 125, no. 3, pp. 282-289, 2003.

[49] P. W. Tse and D. Wang, "The design of a new sparsogram for fast bearing fault diagnosis: part 1 of the two related manuscripts that have a joint title as 'Two automatic vibrationbased fault diagnostic methods using the novel sparsity measurement-parts 1 and 2,' Mechanical Systems and Signal Processing, vol. 40, no. 2, pp. 520-544, 2013.

[50] R. Q. Yan, Y. B. Liu, and R. X. Gao, "Permutation entropy: a nonlinear statistical measure for status characterization of rotary machines," Mechanical Systems and Signal Processing, vol. 29, no. 5, pp. 474-484, 2012.

[51] J. Antoni, "The infogram: Entropic evidence of the signature of repetitive transients," Mechanical Systems and Signal Processing, vol. 74, pp. 73-94, 2016.

[52] L. Zhang, G. Xiong, and W. Huang, "New procedure and index for the parameter optimization of complex wavelet based resonance demodulation," vol. 51, no. 3, pp. 129-138, 2015. 


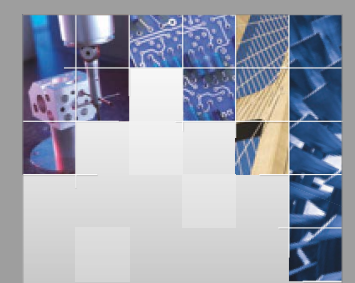

\section{Enfincering}
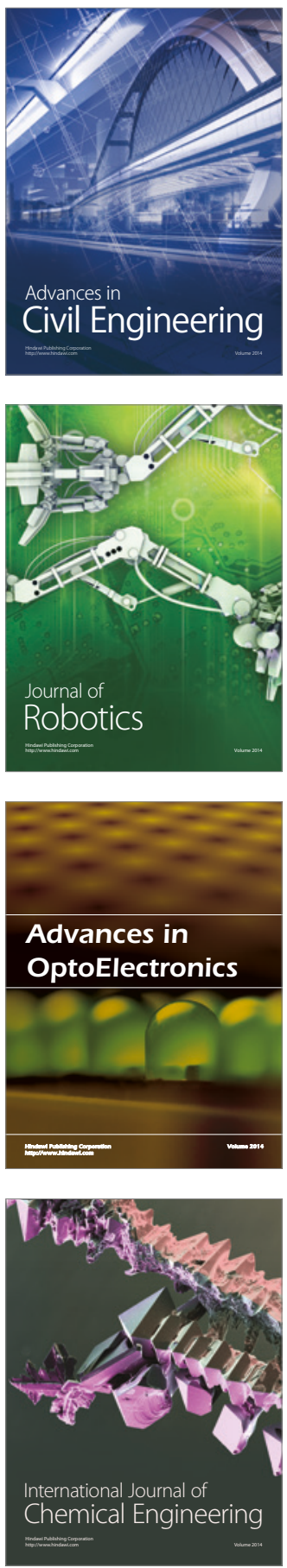

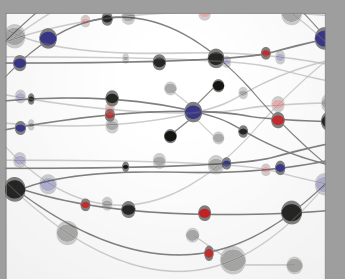

The Scientific World Journal

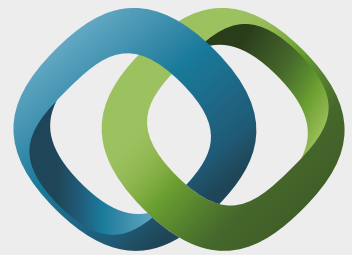

\section{Hindawi}

Submit your manuscripts at

https://www.hindawi.com
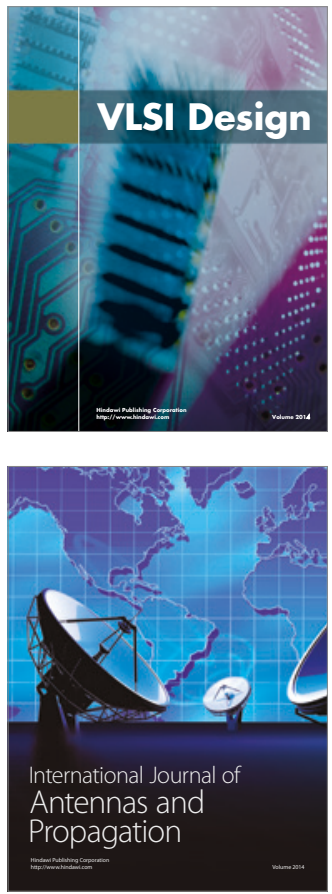

\section{Rotating}

Machinery
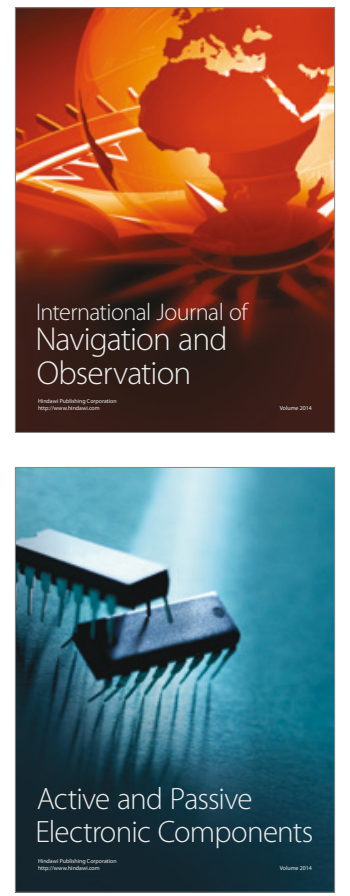
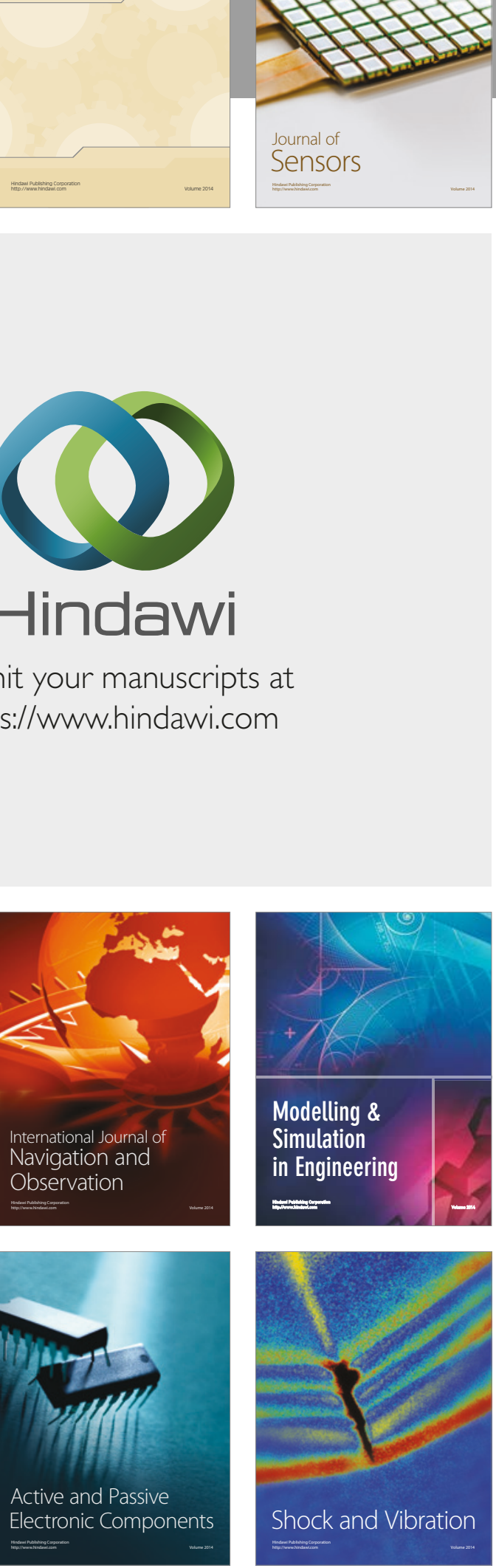
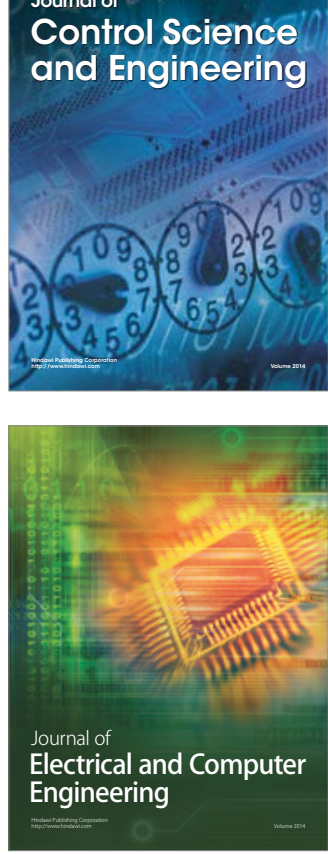

Distributed

Journal of

Control Science

and Engineering
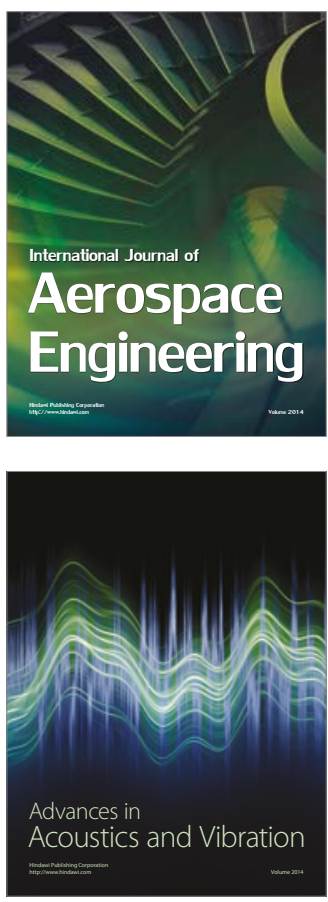

Sensor Networks 